
\title{
$\begin{array}{ll}\text { Research Square } & \begin{array}{l}\text { Preprints are preliminary reports that have not undergone peer review. } \\ \text { They should not be considered conclusive, used to inform clinical practice, } \\ \text { or referenced by the media as validated information. }\end{array}\end{array}$
}

\section{A Prospective and Nutrition-Related Nomogram Model for Prognosis in Colorectal Cancer Survival: A Multicenter Cohort Study in China}

Guo-Tian Ruan

Capital Medical University Affiliated Beijing Shijitan Hospital

Kang-Ping Zhang

Capital Medical University Affiliated Beijing Shijitan Hospital

Qi Zhang

Capital Medical University Affiliated Beijing Shijitan Hospital

Xi Zhang

Capital Medical University Affiliated Beijing Shijitan Hospital

Meng Tang

Capital Medical University Affiliated Beijing Shijitan Hospital

Meng-Meng Song

Capital Medical University Affiliated Beijing Shijitan Hospital

Xiao-Wei Zhang

Capital Medical University Affiliated Beijing Shijitan Hospital

Yi-Zhong Ge

Yuying Children's Hospital of Wenzhou Medical College: Wenzhou Medical University Second Affiliated Hospital

Ming Yang

Capital Medical University Affiliated Beijing Shijitan Hospital

Chun-Hua Song

College of Public Health, zhengzhou university

Ming-Hua Cong

Chinese Academy of Medical Sciences Cancer Institute and Hospital: Cancer Hospital Chinese Academy of Medical Sciences

Zeng-Qing Guo

Fujian Provincial Cancer Hospital

Li-Chen Zhu

School of Preclinical Medicine, Guangxi Medical University

Marco Braga

San Raffaele Hospital: IRCCS Ospedale San Raffaele

Tommy E Cederholm

Uppsala University, clinical nutrition and metabolism

Hong-Xia Xu

Army Military Medical University: Army Medical University

Wei Li

Jilin University First Hospital

Chun-Yun Xiao

Baylor scott and white institute for rehabilitation

hanping shi ( $\nabla$ shihp@ccmu.edu.cn )

Capital Medical University Affiliated Beijing Shijitan Hospital https://orcid.org/0000-0003-4514-8693

\section{Research}

Keywords: Colorectal cancer, nomogram, PG-SGA, GLIM, OS

Posted Date: October 9th, 2020

DOl: https://doi.org/10.21203/rs.3.rs-88669/v1

License: (c) (7) This work is licensed under a Creative Commons Attribution 4.0 International License. Read Full License 


\section{Abstract}

Background

Colorectal cancer (CRC) is a common malignancy with high incidence of malnutrition. Increasing evidence showed that nutritional status could be a prognostic indicator of cancer survival. However, there were few prognostic models involving nutrition-related indicators in CRC. This study aimed to develop a nutrition-related prognostic model for CRC survival.

Methods

This multicenter retrospectively primary cohort data included 1,373 CRC patients, and the external validation cohort enrolled other $108 \mathrm{CRC}$ patients. LASSO and multivariate regression analyses were used to select prognostic indicators and develop the nomogram. We performed C-index, calibration curves, and DCA to assess the discrimination, calibration, and applicability. Finally, the indicators of the nomogram model were used as adjustment factors. Multivariate Cox survival analyses were performed to investigate the benefit of the Patient-Generated Subjective Global Assessment (PG-SGA) and Global Leadership Initiative on Malnutrition (GLIM) malnutrition criteria to CRC prognosis.

Results

Five prognostic indicators (TNM stage, radical resection, reduced food intake, activities and function decline, and low albumin) were chosen to develop the nomogram. C-index of the nomogram was 0.74 (95\% $\mathrm{Cl} 0.72-0.77)$ and $0.72(95 \% \mathrm{Cl} 0.62-0.81)$ in the primary cohort and validation cohort, which were higher than other models. Calibration plots and DCA showed that the nomogram model has a good consistency and clinical benefit in predicting CRC survival. Multivariate Cox survival analysis suggested moderate $(P<0.05, \mathrm{HR} 1.32,95 \% \mathrm{Cl} 1.002-1.75)$ and severe malnutrition $(P<0.001, \mathrm{HR} 1.69,95 \% \mathrm{Cl} 1.27-2.25)$ were associated with worse overall survival (OS) in CRC using the PG-SGA criteria. However, there was no prognostic significance in GLIM criteria (all $P>0.05$ ). Finally, stratified analysis suggested that malnourished CRC patients with low albumin synergistically increased the death risk of CRC OS both in PG-SGA and GLIM. ( $P$ for interaction $=0.007$ and 0.004 , respectively)

\section{Conclusion}

This study established a prospective model to predict the OS in CRC patients using nutrition-related indicators. Moreover, we also found that PG-SGA had a better prognostic and predictive efficiency in CRC than GLIM. These findings grant further verification.

\section{Background}

Colorectal cancer (CRC) is one of the most prevalent and malignant neoplasms globally, with high morbidity and mortality. In 2018, the global cancer data showed that CRC ranked third in morbidity and second in mortality.(1) Subsequently, in 2020, the American Cancer Society releases data reported that the morbidity and mortality of CRC ranked third among all malignant neoplasms in the United States, and the trends were consistent between men and women.(2) As for China, the morbidity and mortality of CRC have been increasing year by year.(3) At present, the tumor-node-metastasis (TNM) staging system is still the most practical and widely used cancer classification and prognosis prediction system in CRC. However, this system cannot provide complete prognostic information.(4) As a consequence, it is necessary to find clinicopathological features other than TNM staging that can also affect the prognosis of CRC patients.

Malnutrition (also known as undernutrition) is more prevalent (approximately $30 \%-60 \%$ )(5) in patients with CRC than non-gastrointestinal cancer.(6) This is due to the combined effects of cancer progression, host response to tumors, anti-cancer therapies, and direct effects of intestinal obstruction and malabsorption. $(7,8)$ Malnutrition can also adversely affect some clinical outcomes of cancer patients, such as overall survival (OS) and tolerance to chemotherapy. $(9,10)$

Varadhan et al. reported that cancer could affect the patients' physiology and psychology, particularly in terms of nutritional status.(11) Nutritional status plays an vital role in influencing the clinical outcome and serves as a prognostic indicator of cancer patients. $(10,12)$ Furthermore, nutritional status affects short term survival of patients, though TNM staging is crucial for accurate prediction of long-term outcome.(13) The combination of them would enhance the accuracy of survival prediction. Therefore, an applicable nutrition assessment tool is necessary to assess nutritional status.

The Patient Generation Subjective Overall Assessment (PG-SGA), adapted from the Subjective Global Assessment, is widely used in clinical and academic research as a reference method for evaluating the nutritional status of cancer patients.(14) It was reported that the PG-SGA has higher clinical benefit in assessing the nutritional status in patients with malignant neoplasms in their digestive system.(15) More specifically, Read et al. found PG-SGA useful as a prognostic model to predict OS in cancer patients. (16) Yang et al. also found that malnutrition was associated with low OS in CRC patients using PG-SGA. (15) Recently, the Global Leadership Initiative on Malnutrition (GLIM) criteria, which served as a new diagnostic tool for malnutrition, was come up and developed.(17) GLIM includes a combination of the phenotype (unintentional weight loss, low body mass index, and reduced muscle loss) and etiology (reduced food intake or assimilation, and acute or chronic inflammation) criteria for diagnosing malnutrition.(18) Previous research has proven the effectiveness of these guidelines in identifying malnutrition.(19) Nevertheless, the effectiveness of GLIM guidelines in cancer patients still needs further verification.(20) Two groups of scientists reported that GLIM-diagnosed malnutrition was an independent risk factor for cancer survival. They also used GLIM to build a prognostic model of cancer.(19) (21) However, the survival prediction of these two nutrition assessment tools in CRC still needs further verification.

The nomogram is an essential part of the decision-making process in modern medical practice. Currently, The nomogram has been extensively used as prognostic models for cancers.(22-24) Therefore, in this study, for the first time, nutrition-related prognostic indicators were incorporated into

Page $2 / 23$ 
clinicopathological prognostic factors (including TNM staging system) to establish a novel prognostic nomogram model. Furthermore, we also compared different established models to screen for the most appliable one. Finally, the survival status of CRC patients with a high incidence of malnutrition was evaluated and predicted.

\section{Methods}

\subsection{Patient screening}

A retrospective and observational analysis was performed in a primary cohort study from multiple Chinese medical centers which collected CRC patients' data from July 2013 to December 2018. The inclusion criteria were: 1) Pathologically diagnosed as primary CRC; 2) Age $\geq 18$ years; 3 ) More than 48 hours of hospitalization; 4) Signed informed consent. The exclusion criteria were: 1) Incomplete clinicopathological parameter information; 2) Age < 18 years; 3) Less than 48 hours of hospitalization; 4) Refused to sign informed consent. Furthermore, a validation cohort data was obtained from the First Affiliated Hospital of Sun Yat-Sen University and the First Affiliated Hospital of The University of Science and Technology of China (USTC) between 2010 and 2019 . The inclusion and exclusion criteria of the verification cohort were consistent with the previous description. Finally, the researchers obtained 1373 patients for the primary cohort and 108 patients for the validation cohort. Fig. 1 presented detailed information for selection in a flowchart. This study followed the Helsinki declaration and was approved by the Institutional Review Board of each hospital. (Registration number: ChiCTR1800020329)

\subsection{Data collection}

\subsubsection{Clinical variable}

This study collected the demographic and clinicopathological characteristic data, including age, sex, lifestyle (smoking status, alcohol consumption, and tea consumption), comorbidity (liver cirrhosis, hypertension, and diabetes, etc.), family history of cancer, nationality, TNM stage, radical resection, nutrition-related information (weight loss, reduced oral intake, activities and function decline, body mass index (BMI), and nutrition support status), total protein and albumin. The weight loss (unintentional) was estimated based on the comparison between the patient's weight at the time of admission (wearing a light hospital gown and without shoes) and the usual weight (the patient's usual measurement and self-description). The reduced intake consisted of reduced food intake and eating problems. It was assessed by comparing the change in food intake between the current and the past month. The eating problems were evaluated by the patient's self-description of the physical symptoms, including nausea, vomiting, diarrhea, constipation, etc. The activities and function decline was estimated by the change within the past month. The BMI calculation formula is as follows: $\mathrm{BMI}=$ body mass $(\mathrm{kilogram}, \mathrm{kg}) / \mathrm{height}(\mathrm{meter}, \mathrm{m})^{2}$.

\subsubsection{Nutrition assessment}

The PG-SGA criteria mainly contained two parts, including the patient's self-assessment section (Box 1: Weight changed in one or six months; Box 2: Food intake changed during the past month; Box 3: Symptoms that affected food intake during the past two weeks; and Box 4: Activities and function changed over the past month) and professional assessment section (Diseases, Metabolic Demand, and Physical Exam). The scored PG-SGA was classified into three categories: A, nomally nourished; B, moderately/suspected malnourished; and C, severely malnourished.

The GLIM criteria consist of two steps: first, we used a nutritional risk screening tool (scored Nutritional Risk Screening-2002, NRS2002) to screen for patients with a risk score of three or greater to classified as at risk of malnutrition; second, malnutrition diagnosis and severity grading based on GLIM criteria. The malnutrition diagnosis involved three phenotypic components (including unintentional weight loss, low BMl, and reduced muscle mass) and two etiological components (reduced food intake and disease burden/inflammation). (Supplemental Table 1) According to the phenotypic criteria, the malnutrition severity grading was classified into Stage 1 (moderately malnourished) and Stage 2 (severely malnourished). (Supplemental Table 2) The reduced muscle mass was accessed by body weight-standardized hand grip strength (HGS/W) and skeletal muscle mass (SMI). The detailed formula as follow: HGS/W= HGS / weight $(\mathrm{kg}) ;(19) \mathrm{SMI}\left(\mathrm{kg} / \mathrm{m}^{2}\right)=$ Appendicular skeletal muscle mass $(\mathrm{ASM}, \mathrm{kg}) /\left[\right.$ height $\left.(\mathrm{m})^{2}\right]=[0.193 \times$ body weight $+0.107 \times$ height $(\mathrm{cm})-4.157 \times$ sex $(\mathrm{men}=1$, women=2) $-0.037 \times$ age -2.631$] /$ [height $\left.(m)^{2}\right] .(25)$

\subsection{Development, validation, and analysis of the nomogram}

Firstly, we used the least absolute shrinkage and selection operator (LASSO) and multivariate Cox regression analyses to screen out and identify the clinically prognostic indicators closely related to the prognosis of CRC in the primary cohort on the R platform (version 3.6.2, http://www.r-proje ct.org/). Then we utilized the selected indicators to construct and develop a prognostic nomogram model. The different models were used to compare the primary and validated cohort, including the nomogram model, TNM stage model, TNM stage + PG-SGA model, and TNM stage + GLIM model. The method of concordance index (Cindex) was performed to measure discriminative ability. The 3-year OS of calibration curves was performed to assess the calibration of the nomogram in both the primary and validated cohort. Finally, to assess and investigate the potential clinical model, the decision curve analysis (DCA) was performed to compare different models to find out the most beneficial model to predict the prognosis of CRC.

\subsection{Survival prediction and stratification analysis based on the nomogram}

To analyze and investigate the survival prediction and clinical benefit of the nomogram model between the PG-SGA and GLIM criteria, the time-dependent receiver operating characteristic (ROC) curves were used to compare different models in the prognostic prediction of the nomogram, PG-SGA, and GLIM. Then we draw a univariate survival curve to analyze the prognostic prediction in CRC between the PG-SGA and GLIM criteria. In addition, we also used the indicators from the constructed prognostic model to adjust the prognostic factors both in the PG-SGA and GLIM criteria, which were based on the nomogram model. Finally, the stratified analysis was performed to evaluate the two malnutrition diagnosis criteria. 


\subsection{Statistical analysis}

Continuous quantitative variables were represented by mean \pm standard deviation, while categorical variables were represented by the number of patients (percentage). We used Student's t-test to analyze continuous variables conforming to a normal distribution, and nonparametric tests (Mann Whitney or Kruskall Wallis) for variables not conforming to normal distribution.

Univariate and multivariate Cox regression were used to analyze the OS of the nomogram model in CRC. We investigated different nutritional assessment criteria for prognosis prediction of CRC survival based on the nomogram model. Hazard ratios (HRs) and $95 \%$ confidence interval (Cl) were used to evaluate the contribution and reduce clinical bias.

The discrimination ability of different models was assessed using Harrell's concordance index (C-index). The calibration measured the difference between predicted and actual survival probability. The time-dependent ROC curve and DCA were performed to estimate the accuracy of survival predictions in the new model and its reliability.

The two tails of $P<0.05$ were indicated to be statistically significant. All the above analyses were performed and detected using SPSS version 20.0 (SPSS Inc, Chicago, IL) and R version 3.6.2.

\section{Results}

\subsection{Clinicopathological characteristics}

In this study, we collected demographic and clinicopathological information from two cohorts. In the primary cohort, a total of 1,373 patients were included, with $58.5 \%$ of them being male $(n=803)$ and $30.7 \%$ being elderly patients $(n=421)$. A median follow-up time was 28.3 months. Based on the PG-SGA criteria, 924 patients (67.3\%) were diagnosed as malnutrition (39.1\% of them being moderately malnourished and $28.2 \%$ being severely malnourished), and 449 patients (32.7\%) were normally nourished. As for the GLIM criteria, 403 patients $(29.4 \%)$ were diagnosed as malnutrition ( $10.2 \%$ of them being moderately malnourished and $19.2 \%$ being severely malnourished), and 970 patients $(70.6 \%)$ were normally nourished.

As for the external validation cohort, a total of 108 patients were included, with $57.4 \%$ of them being male $(n=62)$ and $32.4 \%$ of them being elderly patients $(n=35)$. A median follow-up time was 29.6 months. (Table 1) Based on the PG-SGA criteria, 59 patients $(54.6 \%)$ were diagnosed as malnutrition (29.6\% of them being moderately malnourished and $25 \%$ being severely malnourished), and 49 patients (45.4\%) were normally nourished. As for the GLIM criteria, 43 patients $(39.6 \%)$ were diagnosed as malnutrition ( $9 \%$ of them being moderately malnourished and $30.6 \%$ being severely malnourished), and 65 patients ( $60.2 \%$ ) were normally nourished.

\subsection{Selection of clinical indicators to build the new nomogram model}

We selected the most relevant prognostic indicators through LASSO. The results showed that the indicators of TNM stage, radical resection, reduced food intake, activities and function decline, and low albumin were chosen. (Fig. 2A-B) The multivariate Cox regression analyses suggested that these indicators were associated with the CRC OS and identified as the prognostic indicators for the new nomogram model. (Table 2 and Fig. 2C) Finally, we took advantage of these prognostic indicators to build the prognostic nomogram model of CRC (Fig. 3A) The total points were obtained by adding the scores of each indicator. The more the points, the greater the risk and the lower the probability of survival.

\subsection{Validation and analysis of the nomogram}

We used the $\mathrm{C}$-index and calibration plots to evaluate and investigate the discrimination and calibration of the nomogram. In the primary cohort, the C-index of different models was as follows: nomogram model 0.74 (95\% Cl, 0.72-0.77), TNM stage model 0.70 (95\% Cl, 0.67-0.72), TNM stage + PG-SGA model 0.73 (95\% Cl, 0.70-0.75), and TNM stage + GLIM model 0.71 (95\% Cl, 0.69-0.74). As for the validation cohort, the C-index of different models was as follows: nomogram model $0.72(95 \% \mathrm{Cl}, 0.62-0.81)$, TNM stage model 0.64 (95\% Cl, 0.54-0.74), TNM stage + PG-SGA model 0.68 (95\% Cl, 0.57-0.80), and TNM stage + GLIM model $0.66(95 \% \mathrm{Cl}, 0.56-0.75)$.

The calibration plots were performed and found that the nomogram model exhibited excellent consistency between the primary and validation cohort. (Fig. 3B - C)

Finally, the nomogram model's reliability and benefit were evaluated using DCA, by comparing among the nomogram model, TNM stage model, TNM stage + PG-SGA model, and TNM stage + GLIM model. The results indicated that the nomogram model had a better effort in predicting the 3-year survival of CRC patients. (Fig. 3D)

\subsection{Survival prediction and stratification analysis based on the nomogram}

After successfully constructing the nomogram model, we incorporated it into the nutrition-related prognosis prediction of CRC patients. Firstly, according to the PG-SGA and GLIM criteria, the baseline data was shown in Table 3. Secondly, the 1-, 2-, and 3-year time-dependent ROC curves showed that the area under the curves (AUCs) of the nomogram model (77.7, 78.7, and 73.9, respectively) were more significant than the PG-SGA (62.0, 60.5, and 56.3, respectively) and GLIM model (57.0, 57.0 and 52.7, respectively). (Fig. 4A-C) Then, the univariate survival analysis showed that the malnourished patients had a worse prognosis of CRC OS both in the PG-SGA and GLIM criteria compared to normally-nourished patients $(P<0.05)$. (Fig. 4D-E) Because reduced intake together with activities and function decline were both one of the criteria of the nutritional evaluation tools, they would cause a large bias in the results, so we have removed both when we included the adjusted factors. The prognostic factors of TNM stage, radical resection, and albumin from the nomogram were used to adjust the 
prognostic model between the PG-SGA and GLIM criteria. The multivariate Cox regression analysis demonstrated that the moderately malnourished $(P<0.05$, HR 1.32, 95\% Cl 1.002-1.75) and severely malnourished [ $P<0.001, \mathrm{HR} 1.69,95 \% \mathrm{Cl} 1.27-2.25)$ were associated with lower CRC OS in the PG-SGA criteria. At the same time, there was no prognostic significance in the GLIM criteria (all $P>0.05$ ). (Table 4 and Fig. 5) Finally, we performed a stratification analysis to identify indicators associated with survival in various subgroups. In the PG-SGA criteria, malnourished CRC patients with low albumin synergistically increased the death risk of CRC OS. ( $P$ for interaction $=0.007$ ), and malnourished CRC patients with young age (age $\leq 65$ years), male sex, positive radical resection, and low albumin $(P<0.05)$ were significantly increased the death risk of CRC OS. In the GLIM criteria, malnourished CRC patients with low albumin synergistically increased the death risk of CRC OS ( $P$ for interaction $=0.004)$, and malnourished patients with low albumin was significantly increased the death risk of CRC OS $(P<0.05)$. (Fig. 6)

\section{Discussion}

Accurate prediction of prognosis plays an essential role in the management of CRC as it may help determine the type, timing, and population of treatment.(16) People with different clinicopathological characteristics have different potential prognosis and appropriate treatment strategies.(26) Nutritional status is a crucial factor in predicting cancer risk.(27) To our knowledge, this study aimed to develop a prospective nutrition-related model for predicting the prognosis of CRC by combining clinical and nutritional indicators. In this study, the LASSO and multivariate regression analyses suggested that the TNM stage, radical resection, reduced food intake, activities and function decline, and albumin should be included in the nutritional prognostic model. This study found that these indicators were essential in predicting the CRC prognosis.

Nowadays, the TNM staging system for CRC is a widely used and practical predictor of OS, treatment choice, and recurrence.(28) Although the TNM staging system presents as the gold criteria for predicting the prognosis of CRC, the survival rate varies significantly among patients at the same disease stage, ranging from a few weeks to several years.(29) Yamamoto et al. reported that this might be caused by different prognostic factors, including nutrition status. (30) Furthermore, the TNM stage could only represent the pathological characteristics of tumors. It cannot dynamically detect the prognosis of cancer. Besides, surgery is still the most critical tool for the treatments of solid tumors.(31) However, some patients will develop different degrees of postoperative complications, though $50 \%$ of patients receiving resection could have an improved 5-year OS post-surgery.(32) Reduced food intake together with activities and function decline are essential indicators in evaluating malnutrition, and both could reflect a body's condition. Reduced food intake is a common symptom of cancer patients, caused by a series of metabolic changes or the tumor.(33) Malnutrition is associated with a decline in general functional status, delayed post-surgery recovery, high hospitalization, and increased mortality.(34) As for albumin, previous studies have investigated that albumin is an independent prognostic factor in CRC and hypoalbuminemia was associated with poor survival with CRC. $(8,35,36)$

Nomogram is considered a reliable tool to quantify cancer risk than TNM staging system classification.(37) Previous studies have developed nomograms to predict the clinical outcomes of CRC patients.(38-40) However, some have existed limitations as follows: small sample size, single cohort study, and a lack of validation, etc.(24) We took advantage of these prognostic indicators to build a prognostic nomogram model of CRC. The results indicated that the TNM stage had the most significant contribution to the prognosis of CRC, and the contribution of nutrition-related indicators in this model also had a more significant contribution. Patients with advanced stages of CRC, without radical resection, with reduced food intake, with activities and function decline, and with low albumin got more points, so these patients had a higher risk of death. In this study, the researchers also validated the nomogram using a validation cohort. The primary and validation cohort of nomogram model had the highest C-index ( 0.74 and 0.72 , respectively) and good calibration plots consistency. That was to say, the prognostic predictive ability of the nomogram model was better than other models. Moreover, the nomogram model also showed a better outcome in predicting the 3-year survival of CRC patients when compared to other prognostic models, such as one developed by Valentini et al. from a multicenter clinical trial and Weiser et al. from a retrospective cohort. $(13,39)$

CRC is more likely to cause malnutrition due to its unique characteristics, such as intestinal obstruction. $(7,8)$ The PG-SGA criteria are used as a gold standard for diagnosing cancer malnutrition, which has been widely validated, while the GLIM criteria still need to be further validated. Yin et al. showed $24 \%$ $(292 / 1219)$ of lung cancer (LC) patients have malnutrition according to the GLIM criteria in a cohort study. They found GLIM-diagnosed malnutrition was an independent risk factor for LC prognosis, and severe malnutrition was monotonically related to more LC death $(P=0.002)$.(19) Zhang et al. found that among elderly cancer patients diagnosed as malnourished by the GLIM criteria. For the diagnosis of malnutrition, the primary cohort was approximately $48.4 \%$ (577/1192), and the verification cohort was $46.0 \%$ (138/300). They also found that the GLIM-diagnosed malnutrition (moderate: $P=0.006, \mathrm{HR} 1.35,95 \% \mathrm{Cl}$ 1.09-1.66; and severe: $P<0.001$, HR 1.71, 95\% Cl 1.37-2.1) was significantly associated with shorter OS of elderly cancer patients.(21) Yilmaz et al. found that GLIM-diagnosed malnutrition increased the 1-year mortality of hematologic malignancy. (41) Furthermore, many other reports used the PG-SGA criteria to predict clinical outcomes, such as patient survival, quality of life, postoperative complications, length of stay, and hospital costs.(14) Read et al. showed that PG-SGA could be a good choice to diagnose malnutrition in cancer patients. $(42)$ Many other studies reported similar result. $(14,18,43,44) \mathrm{Gallois}$ et al. collected 168 patients from eight French medical centers and found that PGSGA-diagnosed malnutrition was significantly associated with the OS of metastatic CRC (mCRC) $(P=0.006$, HR 2.6, 1.3-5.3).(45) Similarly, Read et al. found that the PG-SGA criteria had prognostic value in mCRC.(16) Additionally, patients with malnutrition by the PG-SGA criteria indicated a lower survival in advanced CRC.(46)

In this study, compared to the GLIM criteria, the PG-SGA could diagnose more malnourished patients with CRC (primary cohort: $69.4 \%$ vs. $29.4 \%$ and validation cohort: $54.6 \%$ vs. $45.4 \%$ ). we also found that the GLIM criteria could screen out more severely malnourished CRC patients in the primary and validation cohort. This study indicated that the PG-SGA criteria had a better prognostic prediction than the GLIM. Besides, this study showed that the GLIM criteria were not associated with the prognosis for CRC OS, but the PG-SGA were significantly associated. However, the stratified analysis showed that malnourished CRC patients with low albumin had a greater risk of death in both PG-SGA and GLIM. The result was consistent with many others. In short, Albumin might be a good prognostic indicator for malnourished CRC patients, but this result needed to be further confirmed. 
This study also had some limitations: first, the validation data set had a small sample size. Second, some clinical data were not perfect, such as missing some tumor markers and interleukin levels. Finally, this finding needed more validation for the future.

\section{Conclusion}

For the first time, nutrition-related and commonly used clinical parameters were integrated to construct a CRC prognostic nomogram. The nomogram was used to predict CRC survival by the GLIM and the PG-SGA criteria. The nomogram might be a novel and practical tool to evaluate OS for CRC patients.

Moreover, it was worth noting that the PG-SGA malnutrition could serve as a prognostic indicator for CRC and had a better outcome than the GLIM diagnosed malnutrition. Of course, this finding needed further verification.

\section{Declarations}

\section{Consent for publication}

Not applicable.

\section{Data and materials availability}

The datasets used and analyzed during the current study are available from the corresponding author on reasonable request.

\section{Competing interests}

The authors declare that they have no competing interests.

\section{Funding}

This work was supported by the National Key Research and Development Program (2017YFC1309200). 2017YFC1309200 plays an important role in the analysis, interpretation of data and writing the manuscript.

\section{Authors' Contribution}

RGT wrote the manuscript. RGT, ZKP and SHP made substantial contributions to the conception, design and intellectual content of the studies. All authors made key contributions to analysis and interpretation of data. All authors read and approved the final manuscript.

\section{Acknowledgments}

We thank the individuals who participated in this study.

\section{References}

1. Bray F, Ferlay J, Soerjomataram I, Siegel RL, Torre LA, Jemal A. Global cancer statistics 2018: GLOBOCAN estimates of incidence and mortality worldwide for 36 cancers in 185 countries. CA Cancer J Clin. 2018;68(6):394-424.

2. Siegel RL, Miller KD, Jemal A. Cancer statistics, 2020. CA Cancer J Clin. 2020;70(1):7-30.

3. Chen W, Zheng R, Baade PD, Zhang S, Zeng H, Bray F, et al. Cancer statistics in China, 2015. CA Cancer J Clin. 2016;66(2):115-32.

4. Mersakova S, Lasabova Z, Strnadel J, Kalman M, Gabonova E, Sabaka P, et al. Genomic profile and immune contexture in colorectal cancer-relevance for prognosis and immunotherapy. Clin Exp Med. 2020.

5. Lopes JP, de Castro Cardoso Pereira PM, dos Reis Baltazar Vicente AF, Bernardo A, de Mesquita MF. Nutritional status assessment in colorectal cancer patients. Nutr Hosp. 2013;28(2):412-8.

6. Hu WH, Cajas-Monson LC, Eisenstein S, Parry L, Cosman B, Ramamoorthy S. Preoperative malnutrition assessments as predictors of postoperative mortality and morbidity in colorectal cancer: an analysis of ACS-NSQIP. Nutr J. 2015;14:91.

7. Ryan AM, Power DG, Daly L, Cushen SJ, Ni Bhuachalla E, Prado CM. Cancer-associated malnutrition, cachexia and sarcopenia: the skeleton in the hospital closet 40 years later. Proc Nutr Soc. 2016;75(2):199-211.

8. Souza BU, Souza NCS, Martucci RB, Rodrigues VD, Pinho NB, Gonzalez MC, et al. Factors Associated with Sarcopenia in Patients with Colorectal Cancer. Nutr Cancer. 2018;70(2):176-83.

9. Tan CS, Read JA, Phan VH, Beale PJ, Peat JK, Clarke SJ. The relationship between nutritional status, inflammatory markers and survival in patients with advanced cancer: a prospective cohort study. Support Care Cancer. 2015;23(2):385-91.

10. Almasaudi AS, McSorley ST, Dolan RD, Edwards CA, McMillan DC. The relation between Malnutrition Universal Screening Tool (MUST), computed tomography-derived body composition, systemic inflammation, and clinical outcomes in patients undergoing surgery for colorectal cancer. Am J Clin Nutr. 2019;110(6):1327-34.

11. Varadhan KK, Neal KR, Dejong CH, Fearon KC, Ljungqvist O, Lobo DN. The enhanced recovery after surgery (ERAS) pathway for patients undergoing major elective open colorectal surgery: a meta-analysis of randomized controlled trials. Clin Nutr. 2010;29(4):434-40.

12. Maltoni M, Caraceni A, Brunelli C, Broeckaert B, Christakis N, Eychmueller S, et al. Prognostic factors in advanced cancer patients: evidence-based clinical recommendations-a study by the Steering Committee of the European Association for Palliative Care. J Clin Oncol. 2005;23(25):6240-8. 
13. Valentini V, van Stiphout RG, Lammering G, Gambacorta MA, Barba MC, Bebenek M, et al. Nomograms for predicting local recurrence, distant metastases, and overall survival for patients with locally advanced rectal cancer on the basis of European randomized clinical trials. J Clin Oncol. 2011;29(23):316372 .

14. Jager-Wittenaar H, Ottery FD. Assessing nutritional status in cancer: role of the Patient-Generated Subjective Global Assessment. Curr Opin Clin Nutr Metab Care. 2017;20(5):322-9.

15. Yang D, Zheng Z, Zhao Y, Zhang T, Liu Y, Xu X. Patient-generated subjective global assessment versus nutritional risk screening 2002 for gastric cancer in Chinese patients. Future Oncol. 2020;16(3):4475-83.

16. Read JA, Choy ST, Beale PJ, Clarke SJ. Evaluation of nutritional and inflammatory status of advanced colorectal cancer patients and its correlation with survival. Nutr Cancer. 2006;55(1):78-85.

17. Allard JP, Keller H, Gramlich L, Jeejeebhoy KN, Laporte M, Duerksen DR. GLIM criteria has fair sensitivity and specificity for diagnosing malnutrition when using SGA as comparator. Clin Nutr. 2019.

18. De Groot LM, Lee G, Ackerie A, van der Meij BS. Malnutrition Screening and Assessment in the Cancer Care Ambulatory Setting: Mortality Predictability and Validity of the Patient-Generated Subjective Global Assessment Short form (PG-SGA SF) and the GLIM Criteria. Nutrients. 2020;12(8).

19. Yin L, Lin X, Li N, Zhang M, He X, Liu J, et al. Evaluation of the Global Leadership Initiative on Malnutrition Criteria Using Different Muscle Mass Indices for Diagnosing Malnutrition and Predicting Survival in Lung Cancer Patients. JPEN J Parenter Enteral Nutr. 2020.

20. Contreras-Bolivar V, Sanchez-Torralvo FJ, Ruiz-Vico M, Gonzalez-Almendros I, Barrios M, Padin S, et al. GLIM Criteria Using Hand Grip Strength Adequately Predict Six-Month Mortality in Cancer Inpatients. Nutrients. 2019;11(9).

21. Zhang X, Tang M, Zhang Q, Zhang KP, Guo ZQ, Xu HX, et al. The GLIM criteria as an effective tool for nutrition assessment and survival prediction in older adult cancer patients. Clin Nutr. 2020.

22. Sternberg CN. Are nomograms better than currently available stage groupings for bladder cancer? J Clin Oncol. 2006;24(24):3819-20.

23. lasonos A, Schrag D, Raj GV, Panageas KS. How to build and interpret a nomogram for cancer prognosis. J Clin Oncol. 2008;26(8):1364-70.

24. Kawai K, Sunami E, Yamaguchi H, Ishihara S, Kazama S, Nozawa H, et al. Nomograms for colorectal cancer: A systematic review. World J Gastroenterol. 2015;21(41):11877-86.

25. Jensen GL, Cederholm T, Correia M, Gonzalez MC, Fukushima R, Higashiguchi T, et al. GLIM Criteria for the Diagnosis of Malnutrition: A Consensus Report From the Global Clinical Nutrition Community. JPEN J Parenter Enteral Nutr. 2019;43(1):32-40.

26. Che K, Liu F, Wu N, Liu Q, Yuan L, Wei J. Individualized prediction of survival benefits from perioperative chemoradiotherapy for patients with resectable gastric cancer. Cancer Med. 2020.

27. Harvey KB, Moldawer LL, Bistrian BR, Blackburn GL. Biological measures for the formulation of a hospital prognostic index. Am J Clin Nutr. 1981;34(10):2013-22.

28. Chen JH, Zhai ET, Yuan YJ, Wu KM, Xu JB, Peng JJ, et al. Systemic immune-inflammation index for predicting prognosis of colorectal cancer. World J Gastroenterol. 2017;23(34):6261-72.

29. Liang W, Zhang L, Jiang G, Wang Q, Liu L, Liu D, et al. Development and validation of a nomogram for predicting survival in patients with resected nonsmall-cell lung cancer. J Clin Oncol. 2015;33(8):861-9.

30. Yamamoto M, Saito H, Uejima C, Tanio A, Tada Y, Matsunaga T, et al. Combination of Serum Albumin and Cholinesterase Levels as Prognostic Indicator in Patients ith Colorectal Cancer. Anticancer Res. 2019;39(2):1085-90.

31. Sun K, Chen S, Xu J, Li G, He Y. The prognostic significance of the prognostic nutritional index in cancer: a systematic review and meta-analysis. J Cancer Res Clin Oncol. 2014;140(9):1537-49.

32. McArdle CS, Hole DJ. Outcome following surgery for colorectal cancer: analysis by hospital after adjustment for case-mix and deprivation. Br J Cancer. 2002;86(3):331-5.

33. Arends J, Bachmann P, Baracos V, Barthelemy N, Bertz H, Bozzetti F, et al. ESPEN guidelines on nutrition in cancer patients. Clin Nutr. 2017;36(1):11-48.

34. Ahmed T, Haboubi N. Assessment and management of nutrition in older people and its importance to health. Clin Interv Aging. 2010;5:207-16.

35. Wei Y, Xu H, Dai J, Peng J, Wang W, Xia L, et al. Prognostic Significance of Serum Lactic Acid, Lactate Dehydrogenase, and Albumin Levels in Patients with Metastatic Colorectal Cancer. Biomed Res Int. 2018;2018:1804086.

36. Gonzalez-Trejo S, Carrillo JF, Carmona-Herrera DD, Baz-Gutierrez P, Herrera-Goepfert R, Nunez G, et al. Baseline serum albumin and other common clinical markers are prognostic factors in colorectal carcinoma: A retrospective cohort study. Medicine (Baltimore). 2017;96(15):e6610.

37. Fujino S, Myoshi N, Saso K, Sasaki M, Ishikawa S, Takahashi Y, et al. The inflammation-nutrition score supports the prognostic prediction of the TNM stage for colorectal cancer patients after curative resection. Surg Today. 2020;50(2):163-70.

38. Kawai K, Nozawa H, Hata K, Kiyomatsu T, Tanaka T, Nishikawa T, et al. Nomogram Predicting Survival After Recurrence in Patients With Stage I to III Colon Cancer: A Nationwide Multicenter Study. Dis Colon Rectum. 2018;61(9):1053-62.

39. Weiser MR, Landmann RG, Kattan MW, Gonen M, Shia J, Chou J, et al. Individualized prediction of colon cancer recurrence using a nomogram. J Clin Oncol. 2008;26(3):380-5.

40. Peng J, Ding Y, Tu S, Shi D, Sun L, Li X, et al. Prognostic nomograms for predicting survival and distant metastases in locally advanced rectal cancers. PLoS One. 2014;9(8):e106344.

41. Yilmaz M, Atilla FD, Sahin F, Saydam G. The effect of malnutrition on mortality in hospitalized patients with hematologic malignancy. Support Care Cancer. 2020;28(3):1441-8.

Page $7 / 23$ 
42. Read JA, Crockett N, Volker DH, MacLennan P, Choy ST, Beale P, et al. Nutritional assessment in cancer: comparing the Mini-Nutritional Assessment (MNA) with the scored Patient-Generated Subjective Global Assessment (PGSGA). Nutr Cancer. 2005;53(1):51-6.

43. Van Cutsem E, Arends J. The causes and consequences of cancer-associated malnutrition. Eur J Oncol Nurs. 2005;9 Suppl 2:S51-63.

44. Hakonsen SJ, Pedersen PU, Bath-Hextall F, Kirkpatrick P. Diagnostic test accuracy of nutritional tools used to identify undernutrition in patients with colorectal cancer: a systematic review. JBI Database System Rev Implement Rep. 2015;13(4):141-87.

45. Gallois C, Artru P, Lievre A, Auclin E, Lecomte T, Locher C, et al. Evaluation of two nutritional scores' association with systemic treatment toxicity and survival in metastatic colorectal cancer: an AGEO prospective multicentre study. Eur J Cancer. 2019;119:35-43.

46. Gupta D, Lammersfeld CA, Vashi PG, Burrows J, Lis CG, Grutsch JF. Prognostic significance of Subjective Global Assessment (SGA) in advanced colorectal cancer. Eur J Clin Nutr. 2005;59(1):35-40.

\section{Tables}

Table 1 Demographic and Clinicopathological Characteristics of the Primary Cohort and validation cohorts 


\begin{tabular}{|c|c|c|c|c|}
\hline \multirow[t]{2}{*}{ Demographic or clinicopathological characteristic } & \multicolumn{2}{|c|}{ Primary cohort $(\mathrm{N}=1373)$} & \multicolumn{2}{|c|}{ Validation cohort $(\mathrm{N}=108)$} \\
\hline & No. of patients & $\%$ & No. of patients & $\%$ \\
\hline \multicolumn{5}{|l|}{ General information } \\
\hline Sex, male, $\mathrm{n}$ & 803 & 58.5 & 62 & 57.4 \\
\hline Age, $>65$ years, $n$ & 421 & 30.7 & 35 & 32.4 \\
\hline Smoking, yes, $\mathrm{n}$ & 517 & 37.7 & 36 & 33.3 \\
\hline Alcohol consumption, yes, $\mathrm{n}$ & 251 & 18.3 & 18 & 16.7 \\
\hline Tea consumption, yes, $n$ & 374 & 27.2 & 45 & 41.7 \\
\hline \multicolumn{5}{|l|}{ Comorbid disease $(s)^{\star}, \mathrm{n}$} \\
\hline 0 & 877 & 63.9 & 69 & 63.9 \\
\hline 1 & 377 & 27.5 & 30 & 27.7 \\
\hline 2 & 95 & 6.9 & 9 & 8.3 \\
\hline 3 or more & 24 & 1.7 & 1 & 0.9 \\
\hline Family history of cancer, yes, $n$ & 194 & 14.1 & 17 & 15.7 \\
\hline Nationality, Han nationality, yes, $\mathrm{n}$ & 1333 & 97.1 & 102 & 94.4 \\
\hline \multicolumn{5}{|l|}{ TNM stage, $\mathrm{n}$} \\
\hline I & 99 & 7.2 & 8 & 7.4 \\
\hline II & 344 & 25.1 & 34 & 31.5 \\
\hline III & 441 & 32.1 & 41 & 38.0 \\
\hline IV & 489 & 35.6 & 25 & 23.1 \\
\hline Radical resection, yes, $n$ & 539 & 39.3 & 59 & 54.6 \\
\hline \multicolumn{5}{|l|}{ Nutrition related information } \\
\hline \multicolumn{5}{|l|}{ PG-SGA Criteria (nutritional index), $\mathrm{n}$} \\
\hline Normally nourished (1-3) & 449 & 32.7 & 49 & 45.4 \\
\hline Moderately malnourished (4-8) & 537 & 39.1 & 32 & 29.6 \\
\hline Severely malnourished (>9) & 387 & 28.2 & 27 & 25.0 \\
\hline \multicolumn{5}{|l|}{ GLIM Criteria, $n$} \\
\hline Normally nourished & 970 & 70.6 & 65 & 60.2 \\
\hline Moderately malnourished & 140 & 10.2 & 10 & 9 \\
\hline Severely malnourished & 263 & 19.2 & 33 & 30.6 \\
\hline Weight loss, yes, n & 772 & 56.2 & 33 & 30.6 \\
\hline Reduce intake, yes, $n$ & 777 & 56.6 & 66 & 61.1 \\
\hline Actives and function decline, yes, $\mathrm{n}$ & 473 & 34.5 & 25 & 23.1 \\
\hline \multicolumn{5}{|l|}{$\mathrm{BMI}, \mathrm{kg} / \mathrm{m}^{2}, \mathrm{n}$} \\
\hline$<18.5$ & 153 & 11.1 & 18 & 16.7 \\
\hline 18.5-23.9 & 770 & 56.1 & 57 & 52.8 \\
\hline $24-27.9$ & 367 & 26.7 & 26 & 24.1 \\
\hline$\geq 28$ & 332 & 24.1 & 7 & 6.5 \\
\hline Nutritional support, yes, $\mathrm{n}$ & 334 & 24.3 & 59 & 54.6 \\
\hline Total protein, abnormal(low), n & 263 & 28.8 & 18 & 16.7 \\
\hline Albumin, abnormal(low), n & 373 & 19.2 & 20 & 18.5 \\
\hline
\end{tabular}

Notes: TNM stage: Tumor-node-metastasis stage; PG-SGA: Patient-Generated Subjective Global Assessment; GLIM: Global Leadership Initiative on Malnutrition; BMI: Body mass index. 
Table 2 Multivariate Cox regression analysis of the interaction between predictors and overall survival

\begin{tabular}{|c|c|c|c|c|c|c|c|c|}
\hline \multirow[t]{2}{*}{ Variable } & \multicolumn{2}{|l|}{ Model 1} & \multicolumn{2}{|l|}{ Model 2} & \multicolumn{2}{|l|}{ Model 3} & \multicolumn{2}{|l|}{ Model 4} \\
\hline & $\mathrm{HR}(95 \% \mathrm{Cl})$ & $\begin{array}{l}\text { Adjusted } \\
\mathrm{P}\end{array}$ & $\mathrm{HR}(95 \% \mathrm{Cl})$ & $\begin{array}{l}\text { Adjusted } \\
\mathrm{P}\end{array}$ & $\mathrm{HR}(95 \% \mathrm{Cl})$ & $\begin{array}{l}\text { Adjusted } \\
\text { P }\end{array}$ & $\mathrm{HR}(95 \% \mathrm{Cl})$ & $\begin{array}{l}\text { Adjusted } \\
\mathrm{P}\end{array}$ \\
\hline \multicolumn{9}{|l|}{ TNM Stage } \\
\hline 1 & Reference & & Reference & & Reference & & Reference & \\
\hline II & $\begin{array}{l}1.29(0.60- \\
2.79)\end{array}$ & 0.515 & $\begin{array}{l}1.39(0.64- \\
3.01)\end{array}$ & 0.400 & $\begin{array}{l}1.38(0.64- \\
2.99)\end{array}$ & 0.411 & $\begin{array}{l}1.35(0.62- \\
2.92)\end{array}$ & 0.445 \\
\hline III & $\begin{array}{l}2.08(1.00- \\
4.32)\end{array}$ & 0.049 & $\begin{array}{l}2.23(1.08- \\
4.62)\end{array}$ & 0.031 & $\begin{array}{l}2.17(1.05- \\
4.50)\end{array}$ & 0.037 & $\begin{array}{l}2.17(1.05- \\
4.50)\end{array}$ & 0.037 \\
\hline IV & $\begin{array}{l}6.00(2.84- \\
12.66)\end{array}$ & $<0.001$ & $\begin{array}{l}7.16(3.53- \\
14.51)\end{array}$ & $<0.001$ & $\begin{array}{l}7.11(3.51- \\
14.41)\end{array}$ & $<0.001$ & $\begin{array}{l}6.93(3.41- \\
14.06)\end{array}$ & $<0.001$ \\
\hline \multicolumn{9}{|l|}{ Radical resection } \\
\hline No & Reference & & & & & & & \\
\hline Yes & $\begin{array}{l}0.85(0.58- \\
1.26)\end{array}$ & 0.426 & & & & & & \\
\hline \multicolumn{9}{|l|}{ Reduce intake } \\
\hline No & Reference & & & & & & & \\
\hline Yes & $\begin{array}{l}1.30(1.02- \\
1.64)\end{array}$ & 0.033 & & & & & & \\
\hline \multicolumn{9}{|l|}{ Actives and function decline } \\
\hline Yes & Reference & & & & & & & \\
\hline No & $\begin{array}{l}0.59(0.47- \\
0.76)\end{array}$ & $<0.001$ & & & & & & \\
\hline \multicolumn{9}{|l|}{ Albumin $(\mathrm{g} / \mathrm{L})$} \\
\hline Normal and high & Reference & & & & & & & \\
\hline low & $\begin{array}{l}1.42(1.12- \\
1.79)\end{array}$ & 0.004 & & & & & & \\
\hline \multicolumn{9}{|l|}{$\begin{array}{l}\text { PG-SGA Criteria (nutritional } \\
\text { index) }\end{array}$} \\
\hline Normally nourished (1-3) & & & & & Reference & & & \\
\hline $\begin{array}{l}\text { Moderately malnourished (4- } \\
\text { 8) }\end{array}$ & & & & & $\begin{array}{l}1.39(1.06- \\
1.83)\end{array}$ & 0.019 & & \\
\hline Severely malnourished (>9) & & & & & $\begin{array}{l}1.92(1.45- \\
253)\end{array}$ & $<0.001$ & & \\
\hline \multicolumn{9}{|l|}{ GLIM Criteria } \\
\hline Normally nourished & & & & & & & Reference & \\
\hline Moderately malnourished & & & & & & & $\begin{array}{l}1.31(0.93- \\
1.85)\end{array}$ & 0.121 \\
\hline Severely malnourished & & & & & & & $\begin{array}{l}1.37(1.05- \\
1.78)\end{array}$ & 0.019 \\
\hline \multicolumn{9}{|l|}{ C-index } \\
\hline Primary cohorts & $\begin{array}{l}0.74(0.72- \\
0.77)\end{array}$ & & $\begin{array}{l}0.70(0.67- \\
0.72)\end{array}$ & & $\begin{array}{l}0.73(0.70- \\
0.75)\end{array}$ & & $\begin{array}{l}0.71(0.69- \\
0.74)\end{array}$ & \\
\hline Validation cohorts & $\begin{array}{l}0.72(0.62- \\
0.81)\end{array}$ & & $\begin{array}{l}0.64(0.54- \\
0.74)\end{array}$ & & $\begin{array}{l}0.68(0.57- \\
0.80)\end{array}$ & & $\begin{array}{l}0.66(0.56- \\
0.75)\end{array}$ & \\
\hline
\end{tabular}

Notes: Model 1: Adjusted by nomogram (TNM stage, radical resection, reduce intake, actives and function decline, and albumin); Model 2: Adjusted by TNM stage; Model 3: Adjusted by TNM stage and PG-SGA; Model 4: Adjusted by TNM stage and GLIM; HR: Hazard ratio; 95\%Cl: 95\% Confidence interval; TNM stage: Tumor-node-metastasis stage; PG-SGA: Patient-Generated Subjective Global Assessment; GLIM: Global Leadership Initiative on Malnutrition; C-index: concordance index.

Table 3 Characteristics of colorectal cancer in a multicenter cohort based on GLIM criteria and PG-SGA criteria 


\begin{tabular}{|c|c|c|c|c|c|c|c|}
\hline \multirow[t]{2}{*}{ Characteristics } & \multicolumn{4}{|c|}{ GLIM Criteria } & \multicolumn{3}{|c|}{ PG-SGA Criteria } \\
\hline & $\begin{array}{l}\text { Normally } \\
\text { nourished } \\
(n=970)\end{array}$ & $\begin{array}{l}\text { Moderately } \\
\text { malnourished }(n=140)\end{array}$ & $\begin{array}{l}\text { Severely } \\
\text { malnourished }(n=263)\end{array}$ & $\begin{array}{l}\mathrm{P} \\
\text { value }\end{array}$ & $\begin{array}{l}\text { Normally } \\
\text { nourished } \\
(n=449)\end{array}$ & $\begin{array}{l}\text { Moderately } \\
\text { malnourished }(n=537)\end{array}$ & $\begin{array}{l}\text { Severely } \\
\text { malnourished }(n=387)\end{array}$ \\
\hline TNM stage & & & & 0.168 & & & \\
\hline I & $79(5.8)$ & $8(0.6)$ & $12(0.9)$ & & $40(2.9)$ & $38(2.8)$ & $21(1.5)$ \\
\hline II & 245(17.8) & $28(2.0)$ & $71(5.2)$ & & 128(9.3) & $124(9.0)$ & $92(6.7)$ \\
\hline III & $316(23.0)$ & $45(3.3)$ & $80(5.8)$ & & 116(8.4) & $202(14.7)$ & 123(9.0) \\
\hline IV & $330(24.0)$ & $59(4.3)$ & 100(7.3) & & 165(12) & 173(12.6) & 151(11) \\
\hline $\begin{array}{l}\text { Radical } \\
\text { resection } \llbracket y e s \\
(n \%)\end{array}$ & $398(29.0)$ & $44(3.2)$ & $97(7.1)$ & 0.064 & 183(13.3) & $230(16.8)$ & $126(9.2)$ \\
\hline $\begin{array}{l}\text { Reduce } \\
\text { intake『yes } \\
(n \%)\end{array}$ & $323(23.5)$ & $89(6.5)$ & $176(12.8)$ & $<0.001$ & $29(2.1)$ & $239(17.4)$ & $320(23.3)$ \\
\hline $\begin{array}{l}\text { Actives and } \\
\text { function } \\
\text { decline冈yes } \\
\text { (n\%) }\end{array}$ & $269(19.6)$ & $66(4.8)$ & 138(10.1) & $<0.001$ & $40(2.9)$ & $182(13.3)$ & 251(18.3) \\
\hline Albumin & $38.65 \pm 4.85$ & $36.83 \pm 5.41$ & $35.76 \pm 6.30$ & $<0.001$ & $39.78 \pm 4.31$ & $38.02 \pm 5.14$ & $35.59 \pm 5.82$ \\
\hline
\end{tabular}

Notes: TNM stage: Tumor-node-metastasis stage; PG-SGA: Patient-Generated Subjective Global Assessment; GLIM: Global Leadership Initiative on Malnutrition.

Table 4 Analysis of the prognostic values of GLIM Criteria and PG-SGA Criteria in colorectal cancer

\begin{tabular}{|lllll|}
\hline Variable & OS* & & \\
& HR $(95 \% \mathrm{Cl})$ & Crude P & HR $(95 \% \mathrm{Cl})$ & Adjusted P \\
\hline GLIM Criteria & & & & \\
\hline Normally nourished & Reference & & Reference & \\
\hline Moderately malnourished & $1.42(1.01-2.00)$ & 0.043 & $1.27(0.90-1.79)$ & 0.174 \\
\hline Severely malnourished & $1.41(1.08-1.832)$ & 0.011 & $1.25(0.96-1.63)$ & 0.104 \\
\hline PG-SGA Criteria & & & & \\
\hline Normally nourished & Reference & & Reference & \\
\hline Moderately malnourished & $1.27(0.96-1.67)$ & 0.093 & $1.32(1.002-1.75)$ & 0.048 \\
\hline Severely malnourished & $1.89(1.43-2.49)$ & $<0.001$ & $1.69(1.27-2.25)$ & $<0.001$ \\
\hline
\end{tabular}

Notes: *: Adjusted by nomogram (TNM stage, radical resection, and albumin); OS: Overall survival; HR: Hazard ratio; $95 \% \mathrm{Cl}$ : $95 \%$ Confidence interval; TNM stage: Tumor-node-metastasis stage; PG-SGA: Patient-Generated Subjective Global Assessment; GLIM: Global Leadership Initiative on Malnutrition.

\section{Figures}


Colorectal cancer patients enrolled in a Chinese multicenter cohort study

$$
(n=1496)
$$

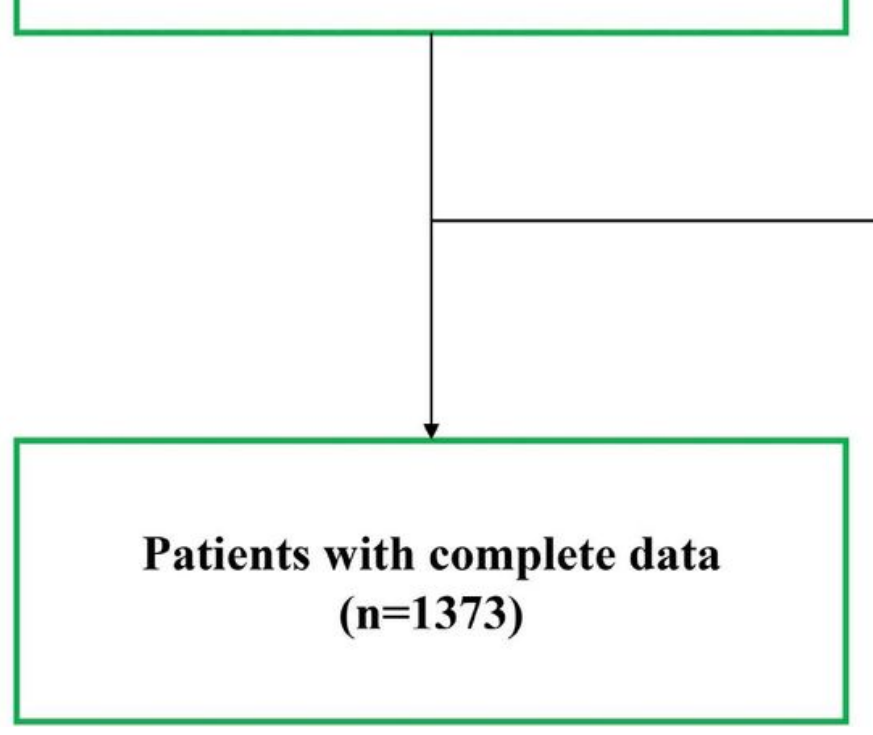

Figure 1

Flowchart of patient selection for this study

Colorectal cancer patients enrolled in a Chinese multicenter cohort study

$$
(n=1496)
$$

Excluded patients $(n=123)$

1. Total protein missing data $(n=13)$

2. Albumin missing data $(n=52)$

3. HGS missing data $(n=58)$

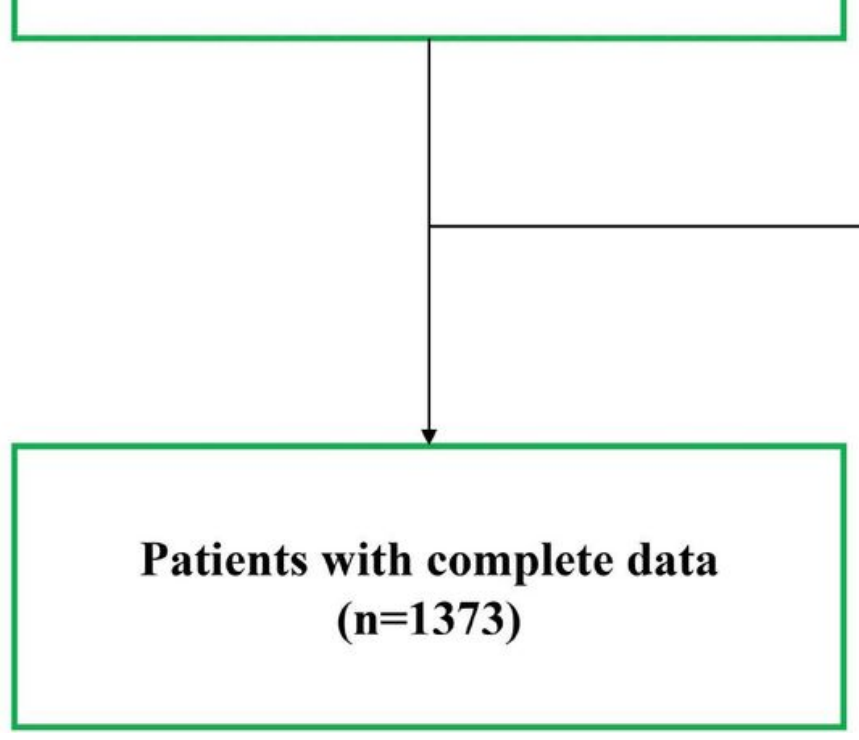

Figure 1

Flowchart of patient selection for this study

Excluded patients $(\mathrm{n}=123)$

1. Total protein missing data $(n=13)$

2. Albumin missing data $(n=52)$

3. HGS missing data $(n=58)$ 


\section{Colorectal cancer patients enrolled in} a Chinese multicenter cohort study

$$
(n=1496)
$$

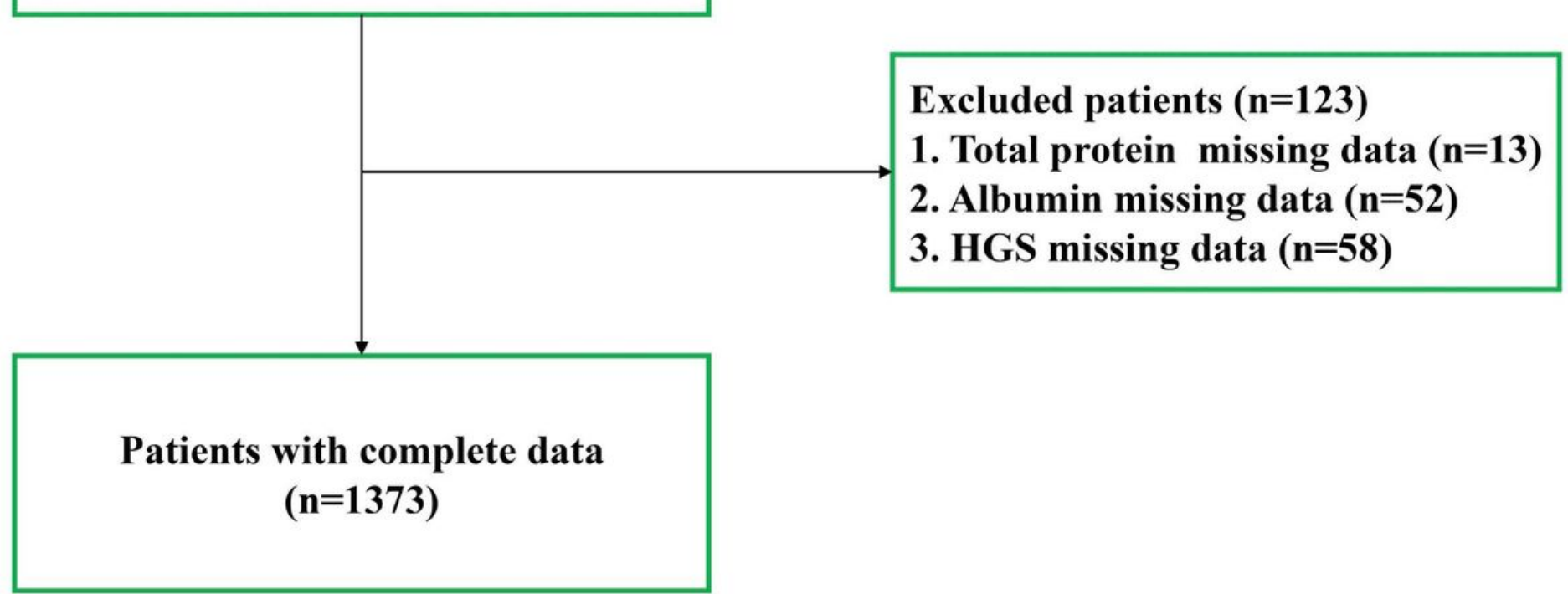

Figure 1

Flowchart of patient selection for this study

A

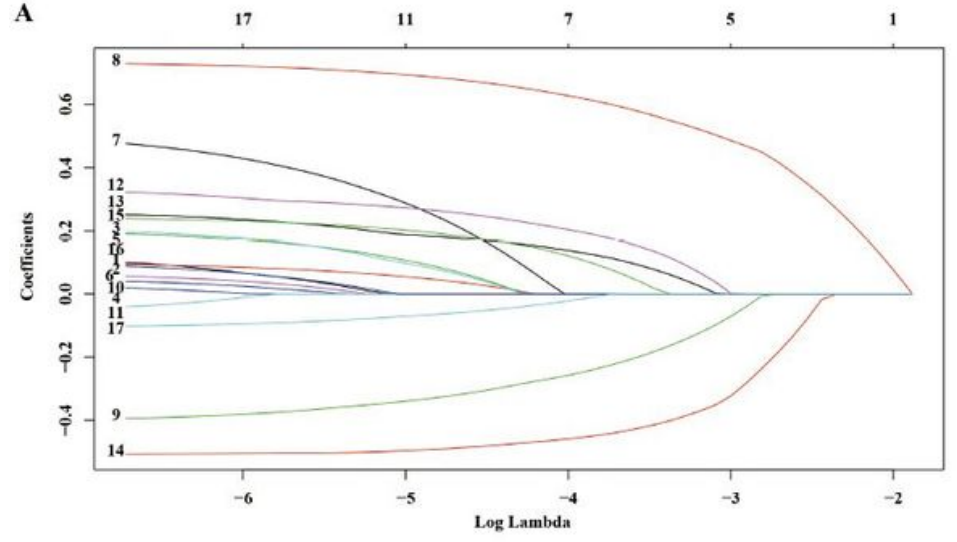

$\begin{array}{lllllllllllllllllllllll}\text { B } & 17 & 17 & 17 & 16 & 15 & 14 & 11 & 11 & 11 & 9 & 7 & 7 & 6 & 6 & 5 & 5 & 3 & 2 & 2 & 1 & 1 & 1\end{array}$

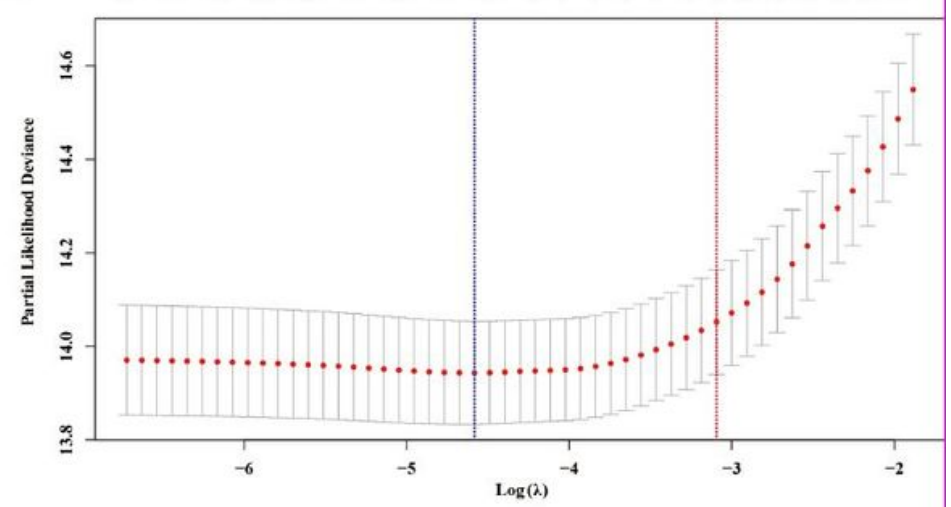

C

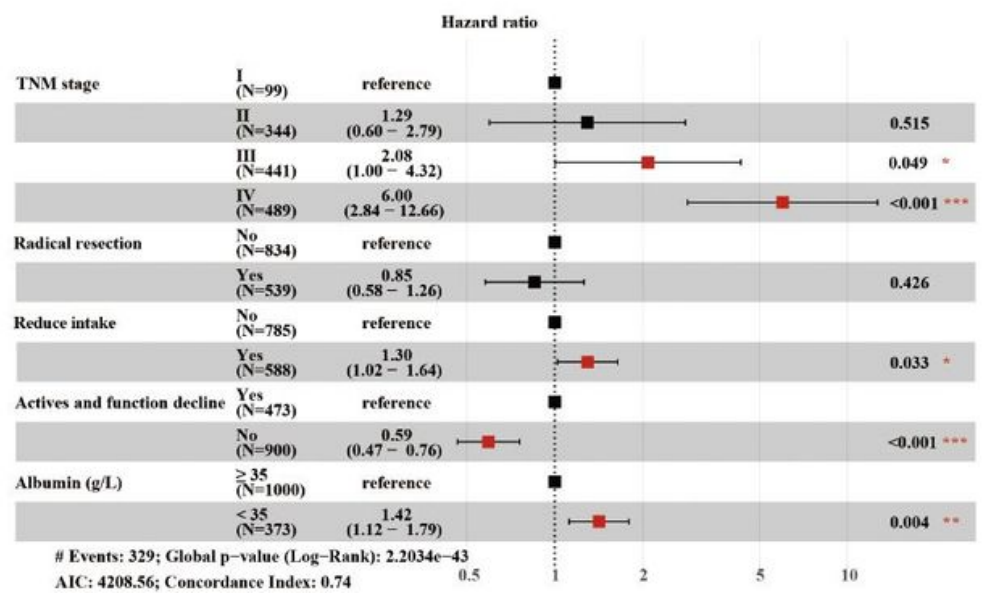

Figure 2

Identification of prognostic indicators in CRC using LASSO and Cox regression analysis. (A) LASSO coefficient profiles of 17 indicators in CRC; (B) Plots of the cross-validation error rates. Each dot represents a lambda value along with error bars to give a confidence interval for the cross-validated error rate; (C) 
Multivariate Cox regression identified five prognostic indicators in the primary cohort. Notes: CRC: Colorectal cancer; LASSO: Least absolute shrinkage and selection operator; TNM stage: Tumor-node-metastasis stage.
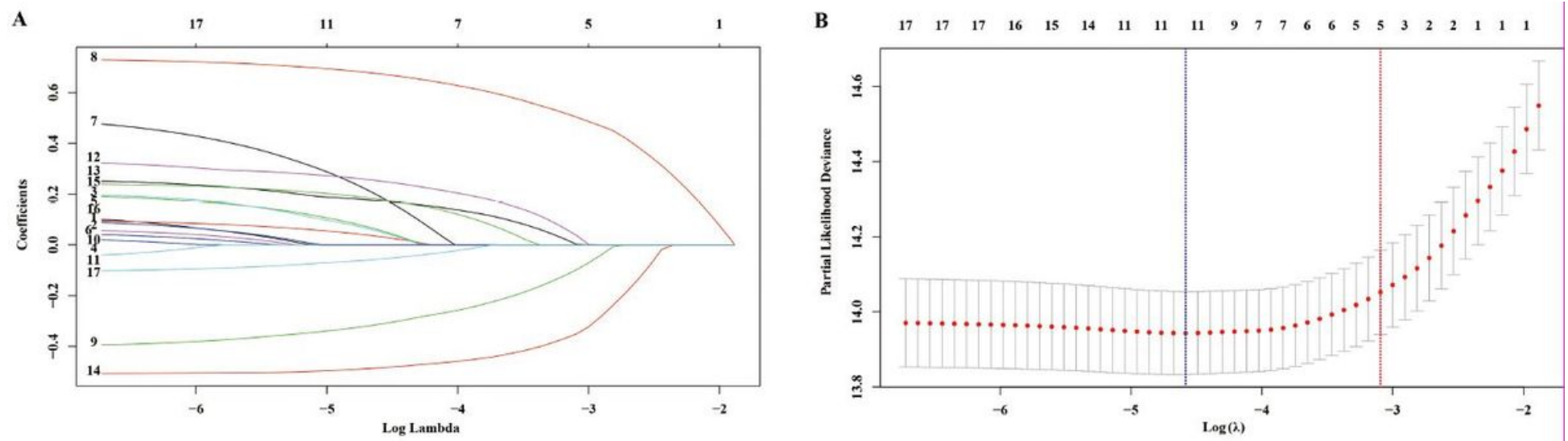

C

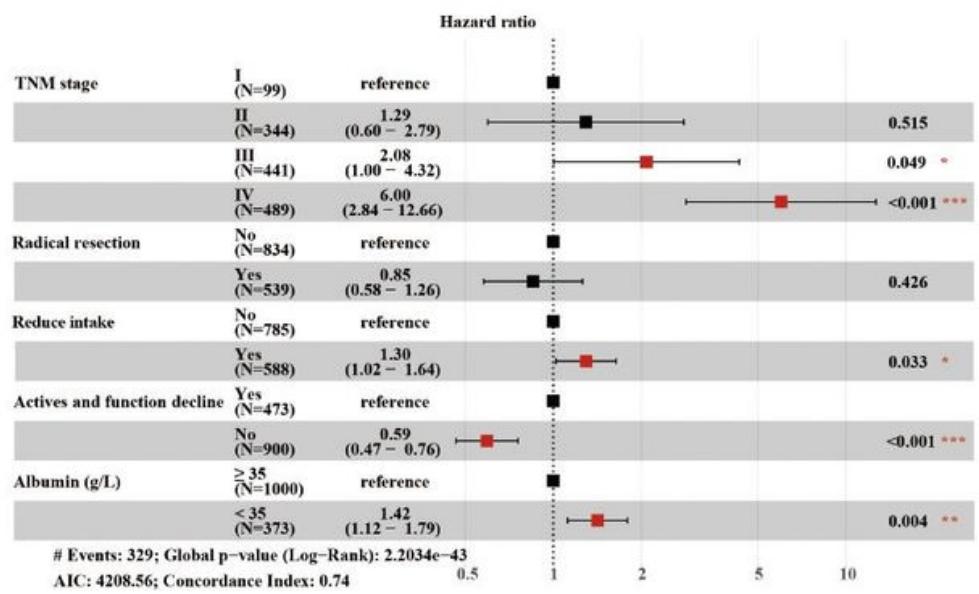

Figure 2

Identification of prognostic indicators in CRC using LASSO and Cox regression analysis. (A) LASSO coefficient profiles of 17 indicators in CRC; (B) Plots of the cross-validation error rates. Each dot represents a lambda value along with error bars to give a confidence interval for the cross-validated error rate; (C) Multivariate Cox regression identified five prognostic indicators in the primary cohort. Notes: CRC: Colorectal cancer; LASSO: Least absolute shrinkage and selection operator; TNM stage: Tumor-node-metastasis stage. 
A

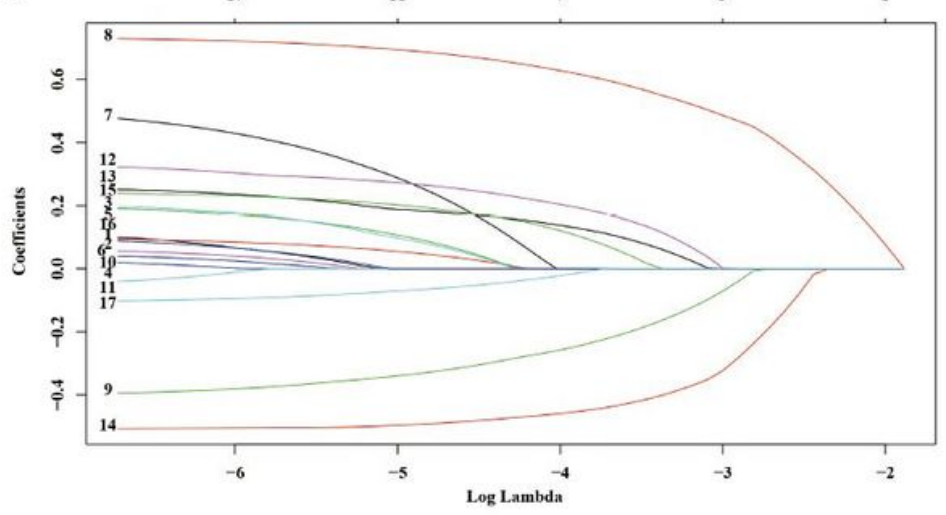

B

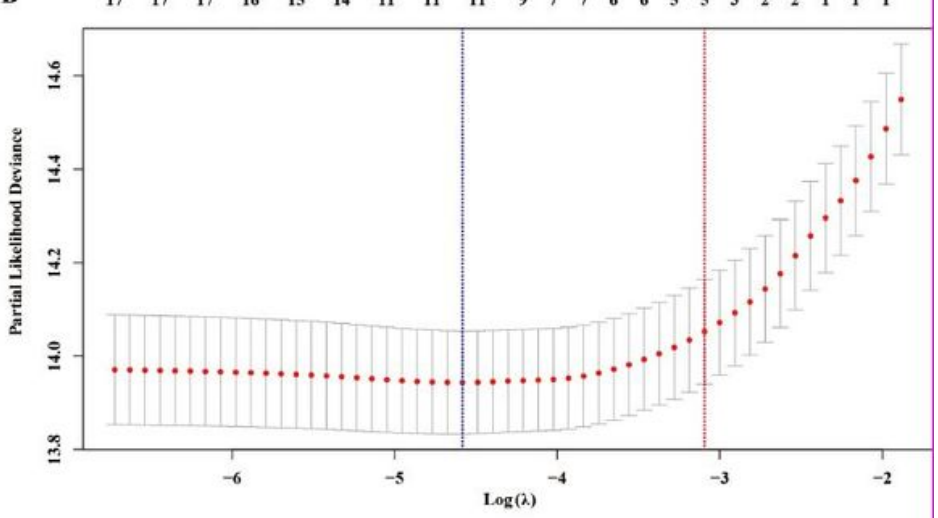

C

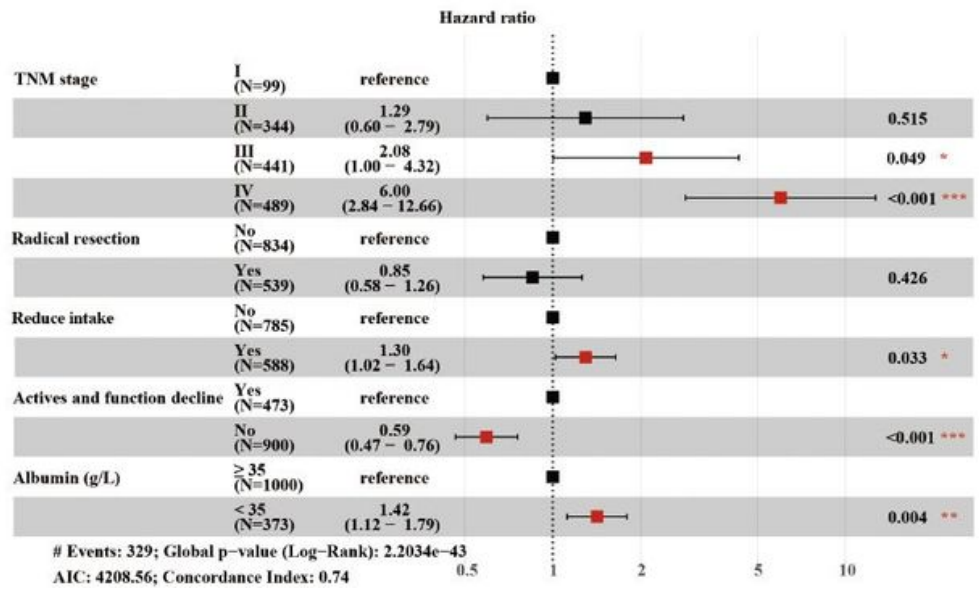

\section{Figure 2}

Identification of prognostic indicators in CRC using LASSO and Cox regression analysis. (A) LASSO coefficient profiles of 17 indicators in CRC; (B) Plots of the cross-validation error rates. Each dot represents a lambda value along with error bars to give a confidence interval for the cross-validated error rate; (C) Multivariate Cox regression identified five prognostic indicators in the primary cohort. Notes: CRC: Colorectal cancer; LASSO: Least absolute shrinkage and selection operator; TNM stage: Tumor-node-metastasis stage. 
TNM stage

Radical resection

Reduce intake

Actives and function decline

Albumin $(\mathrm{g} / \mathrm{L})$

Total Points

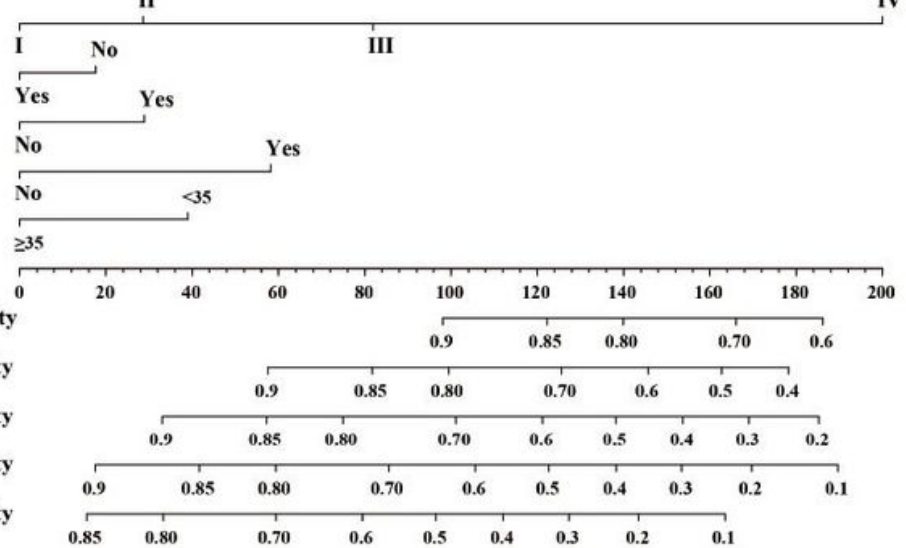

12-Month Survival probability

24-Month survival probability

36-Month survival probability

48-Month survival probability

60-Month survival probability

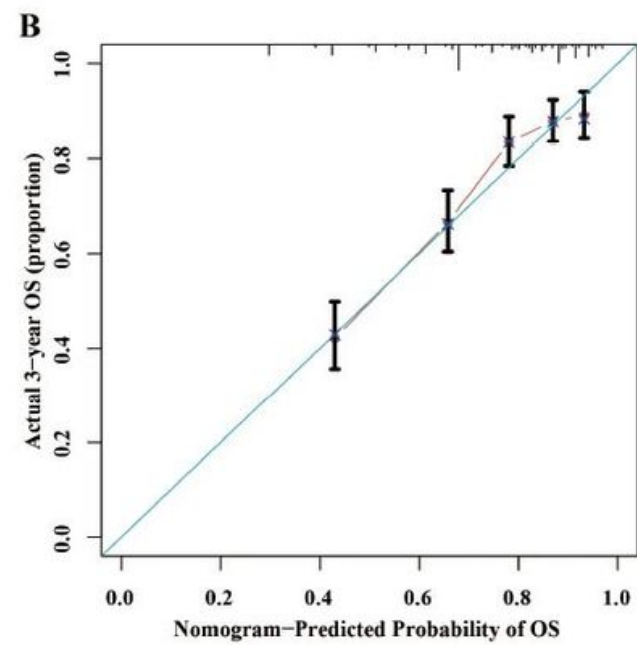

C

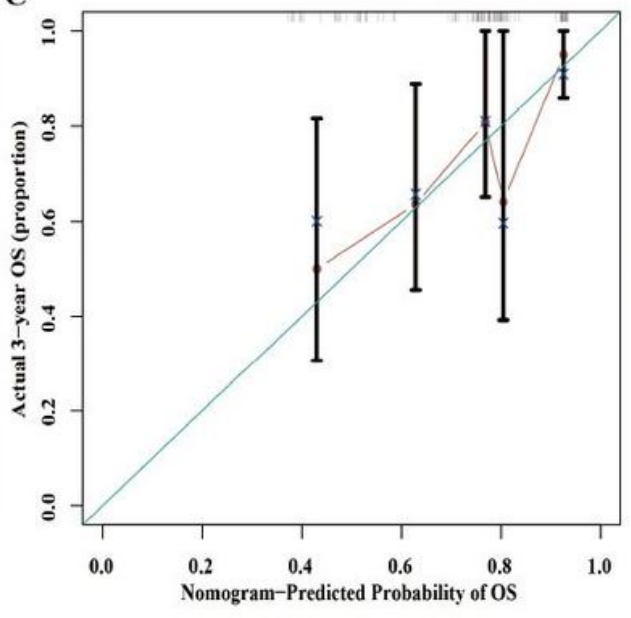

D

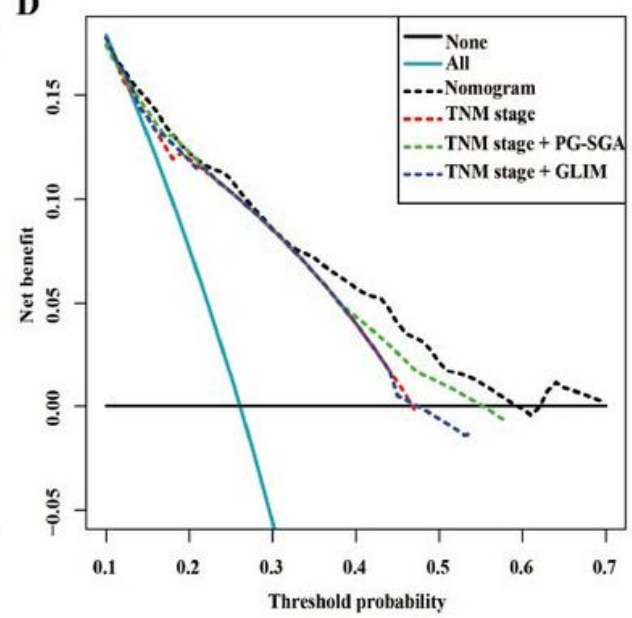

Figure 3

Development, validation, and analysis of the prognostic significanceof the nomogram model in CRC. (A) Prognostic nomogram predicting 1-, 2-, 3-, 4-, and 5year OS probability using the five prognostic indicators. (B) Calibration curve of the nomogram predicting the 3 -year probability of OS in the primary cohort. (C) Calibration curve of the nomogram predicting the 3-year probability of OS in the external validation cohort. (D) DCA of the nomogram model (black line), TNM staging system model (red line), TNM staging system combined with PG-SGA model (green line), and TNM staging system combined with GLIM model (blue line). Notes: CRC: Colorectal cancer; OS: Overall survival; TNM stage: Tumor-node-metastasis stage; PG-SGA: Patient-Generated Subjective Global Assessment; GLIM: Global Leadership Initiative on Malnutrition. 


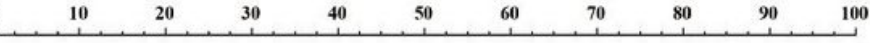

TNM stage

Radical resection

Reduce intake

Actives and function decline

Albumin $(\mathrm{g} / \mathbf{L})$

Total Points

IV

12-Month Survival probability

24-Month survival probability

36-Month survival probability

48-Month survival probability

60-Month survival probability

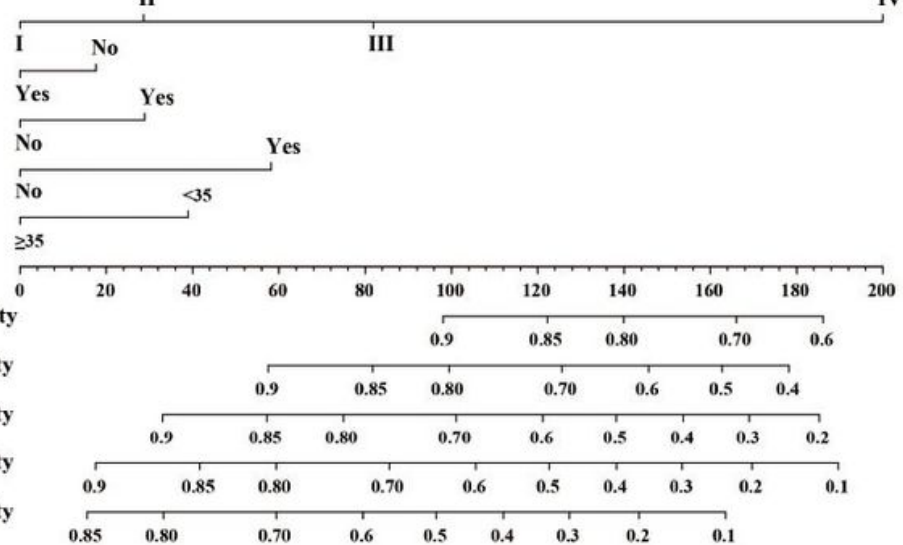

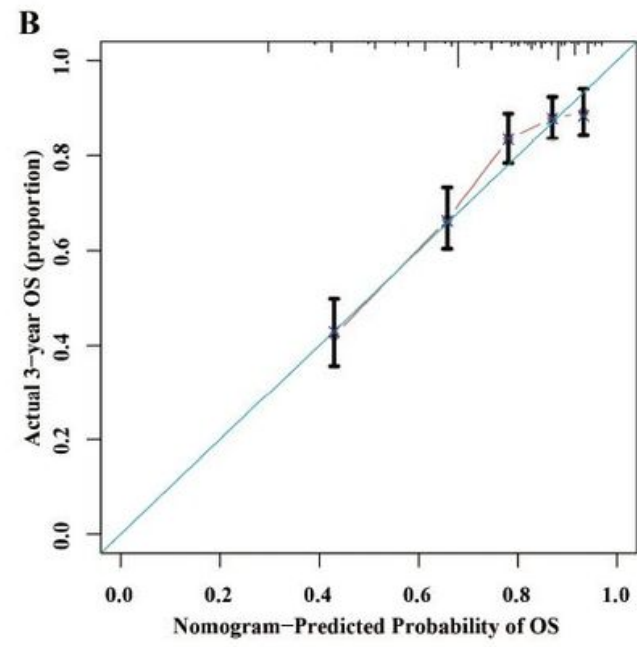

C

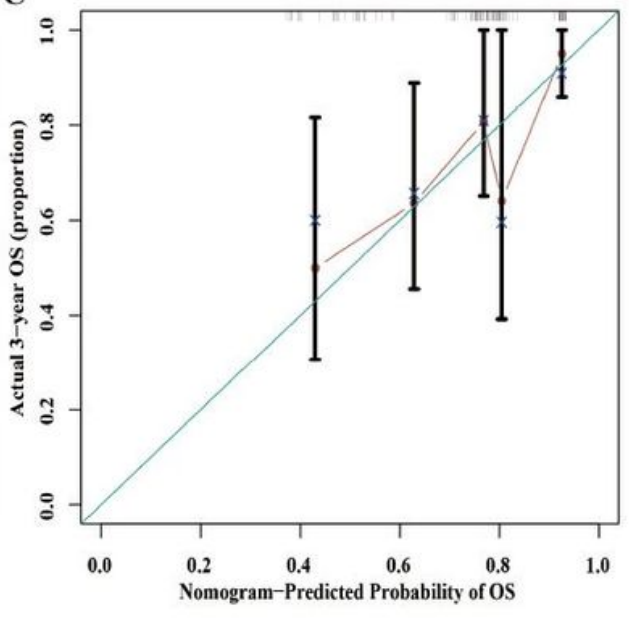

D

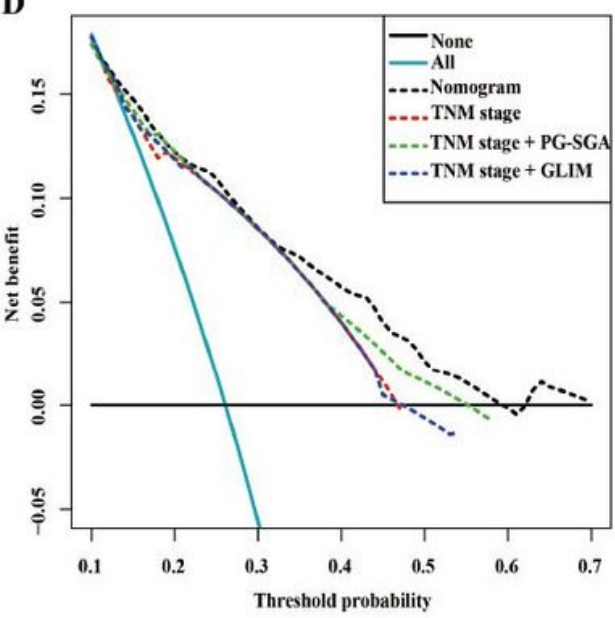

Figure 3

Development, validation, and analysis of the prognostic significanceof the nomogram model in CRC. (A) Prognostic nomogram predicting 1-, 2-, 3-, 4-, and 5year OS probability using the five prognostic indicators. (B) Calibration curve of the nomogram predicting the 3-year probability of OS in the primary cohort. (C) Calibration curve of the nomogram predicting the 3-year probability of OS in the external validation cohort. (D) DCA of the nomogram model (black line), TNM staging system model (red line), TNM staging system combined with PG-SGA model (green line), and TNM staging system combined with GLIM model (blue line). Notes: CRC: Colorectal cancer; OS: Overall survival; TNM stage: Tumor-node-metastasis stage; PG-SGA: Patient-Generated Subjective Global Assessment; GLIM: Global Leadership Initiative on Malnutrition. 


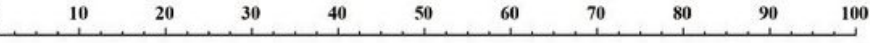

TNM stage

Radical resection

Reduce intake

Actives and function decline

Albumin $(\mathrm{g} / \mathbf{L})$

Total Points

IV

12-Month Survival probability

24-Month survival probability

36-Month survival probability

48-Month survival probability

60-Month survival probability

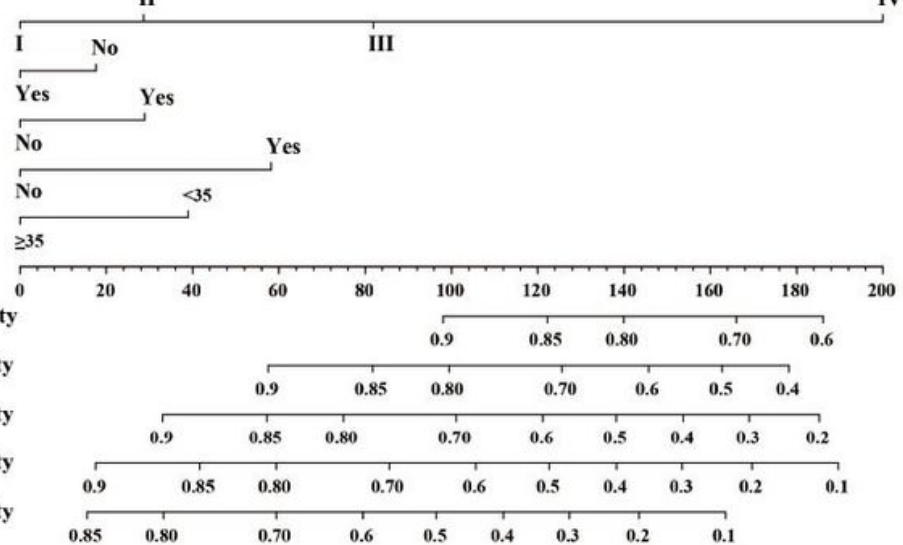

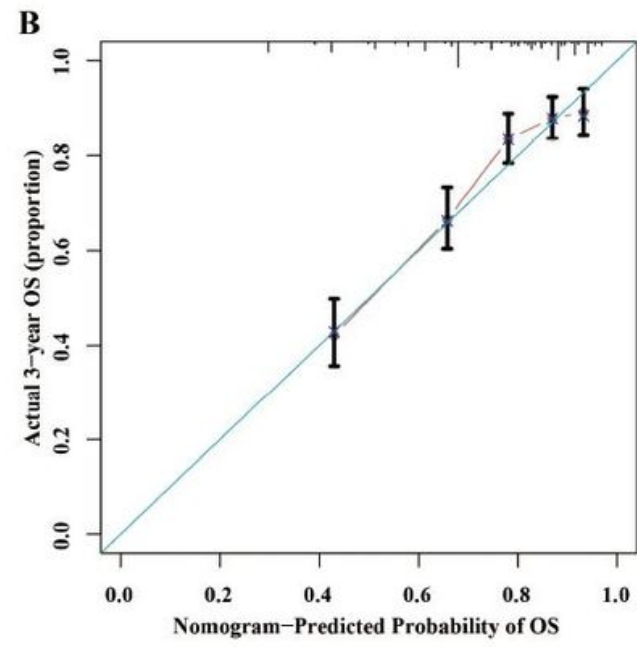

C

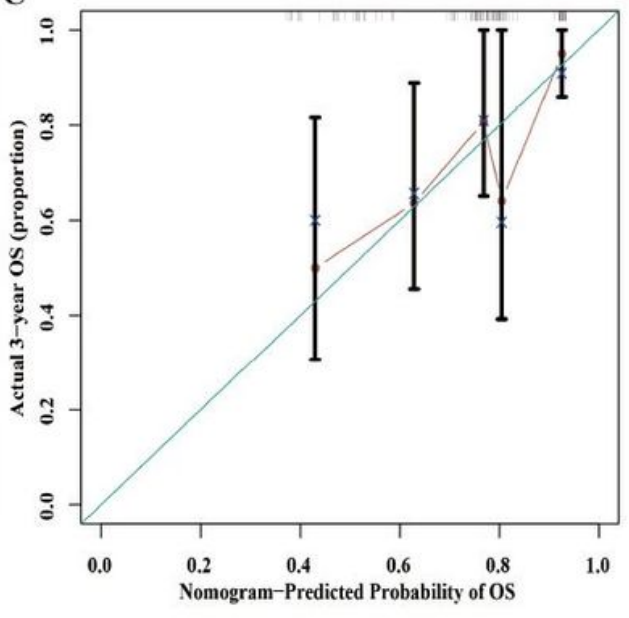

D

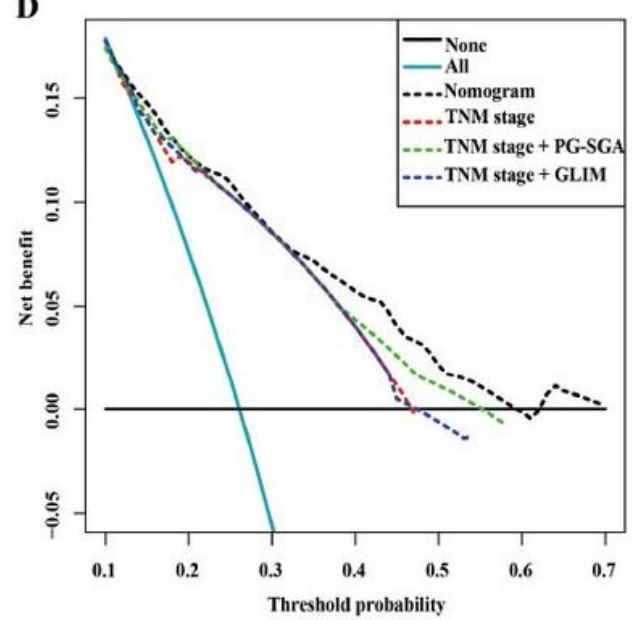

Figure 3

Development, validation, and analysis of the prognostic significanceof the nomogram model in CRC. (A) Prognostic nomogram predicting 1-, 2-, 3-, 4-, and 5year OS probability using the five prognostic indicators. (B) Calibration curve of the nomogram predicting the 3-year probability of OS in the primary cohort. (C) Calibration curve of the nomogram predicting the 3-year probability of OS in the external validation cohort. (D) DCA of the nomogram model (black line), TNM staging system model (red line), TNM staging system combined with PG-SGA model (green line), and TNM staging system combined with GLIM model (blue line). Notes: CRC: Colorectal cancer; OS: Overall survival; TNM stage: Tumor-node-metastasis stage; PG-SGA: Patient-Generated Subjective Global Assessment; GLIM: Global Leadership Initiative on Malnutrition. 


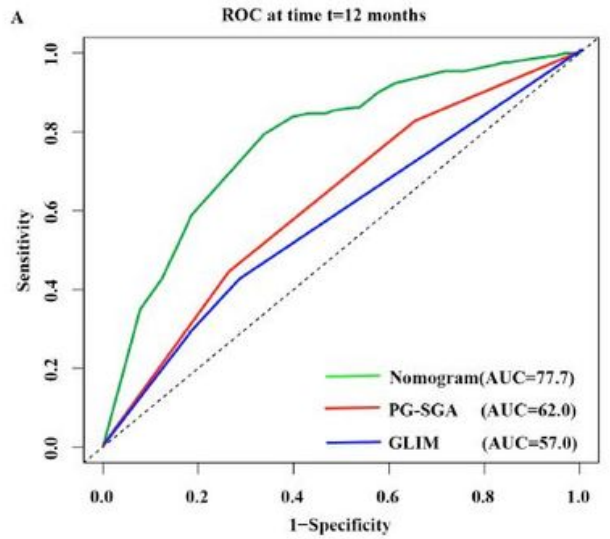

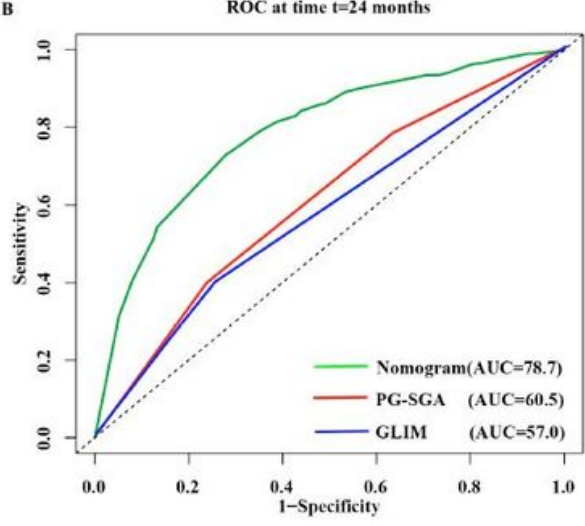

C

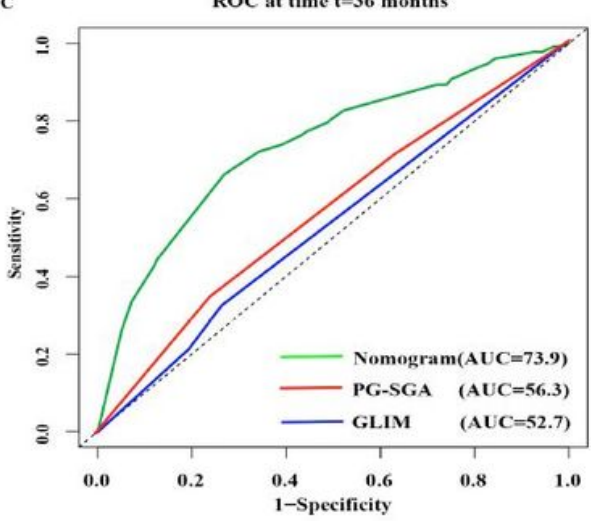

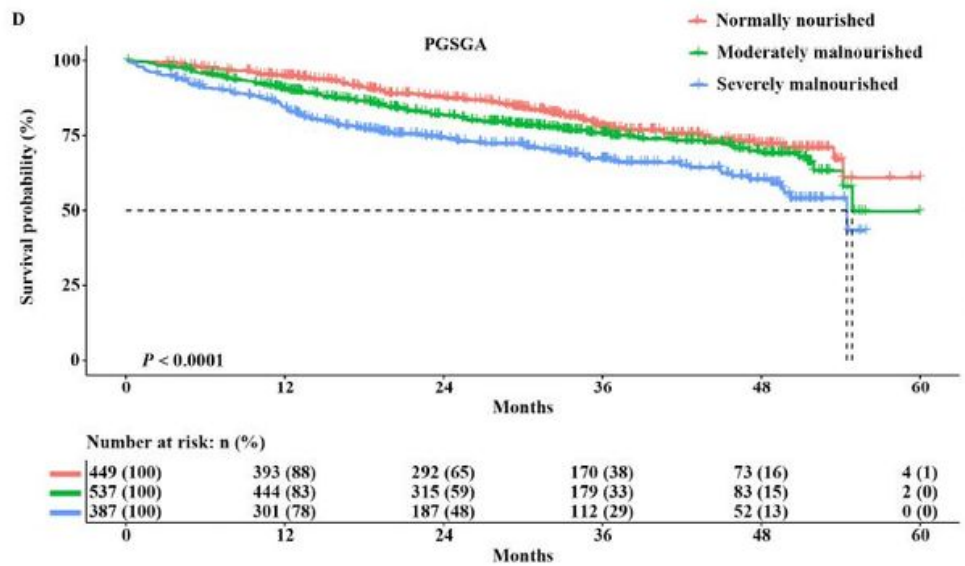

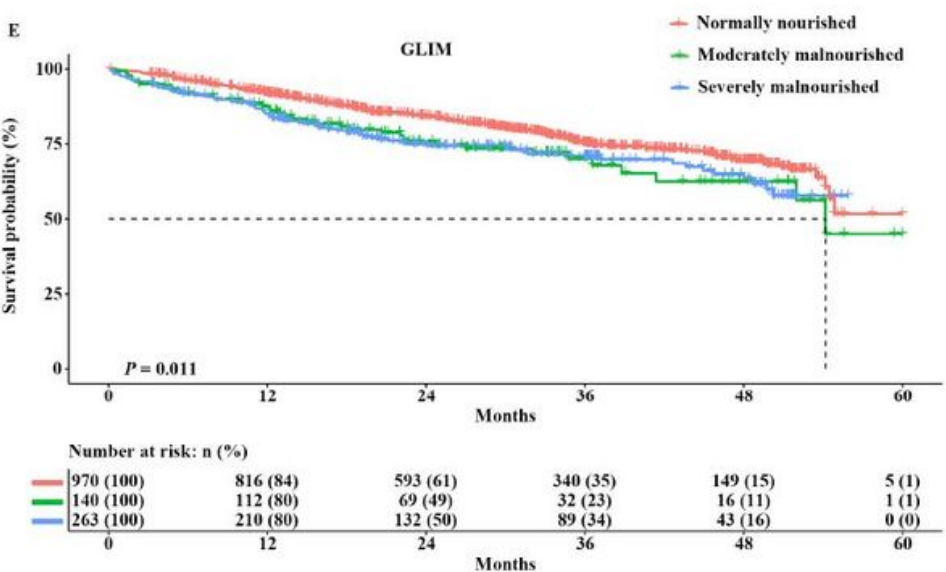

\section{Figure 4}

The 12-, 24-, and 36-month time-dependent ROC curves and survival curves of CRC OS. (A) ROC curves for 12-, 24-, and 36-month OS in the primary cohort. (B) Survival curve of PG-SGA in CRC OS; (C) Survival curve of GLIM in CRC OS. Notes: CRC: Colorectal cancer; OS: Overall survival; PG-SGA: Patient-Generated Subjective Global Assessment; GLIM: Global Leadership Initiative on Malnutrition. 


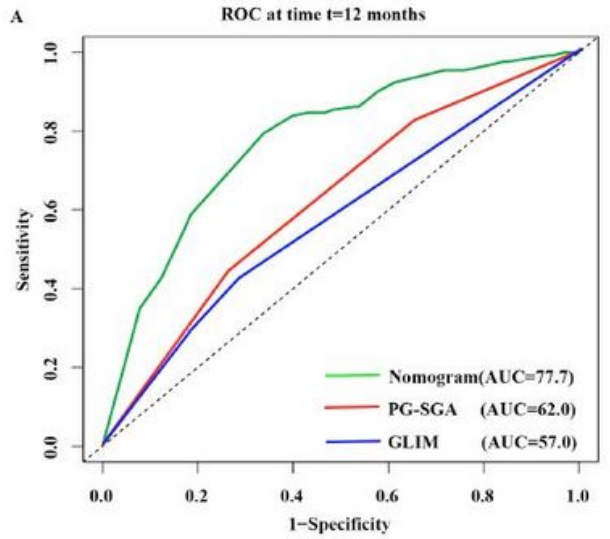

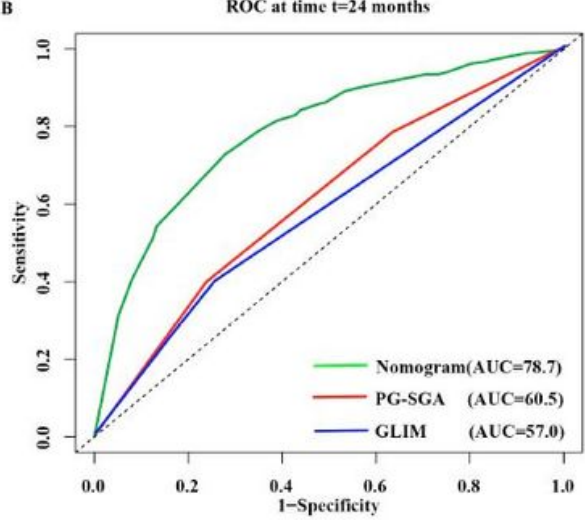

C

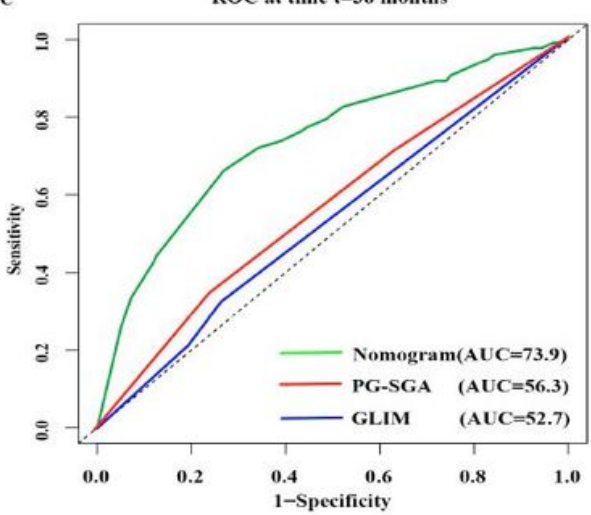

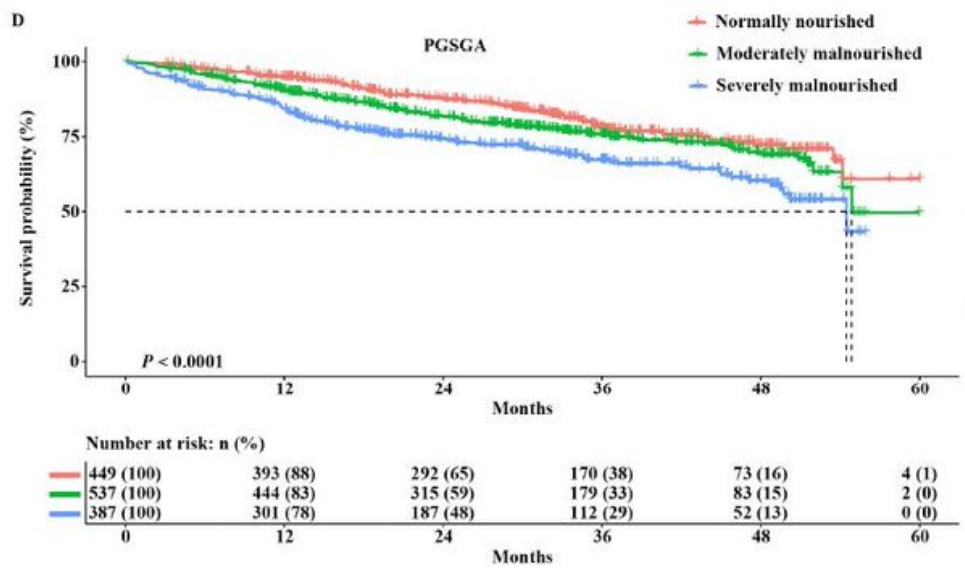

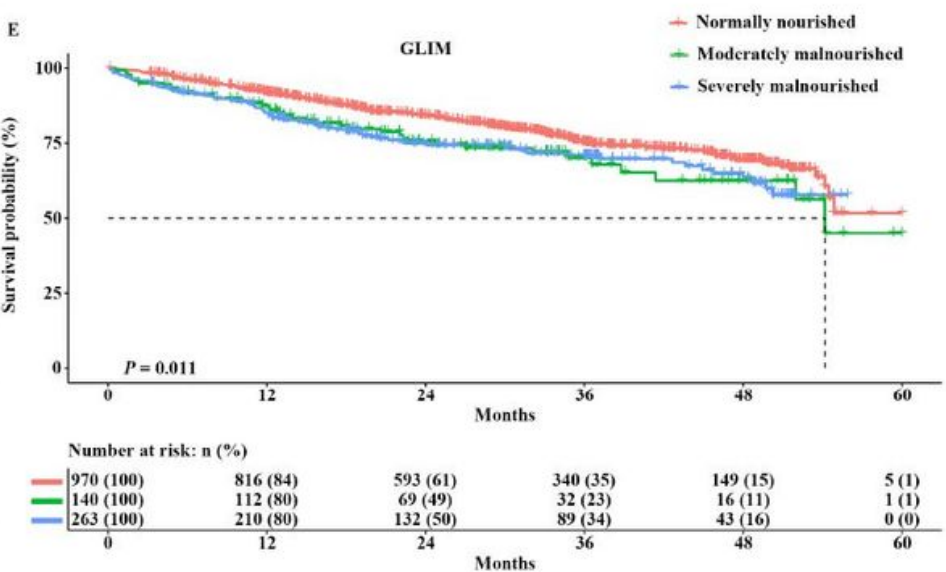

\section{Figure 4}

The 12-, 24-, and 36-month time-dependent ROC curves and survival curves of CRC OS. (A) ROC curves for 12-, 24-, and 36-month OS in the primary cohort. (B) Survival curve of PG-SGA in CRC OS; (C) Survival curve of GLIM in CRC OS. Notes: CRC: Colorectal cancer; OS: Overall survival; PG-SGA: Patient-Generated Subjective Global Assessment; GLIM: Global Leadership Initiative on Malnutrition. 

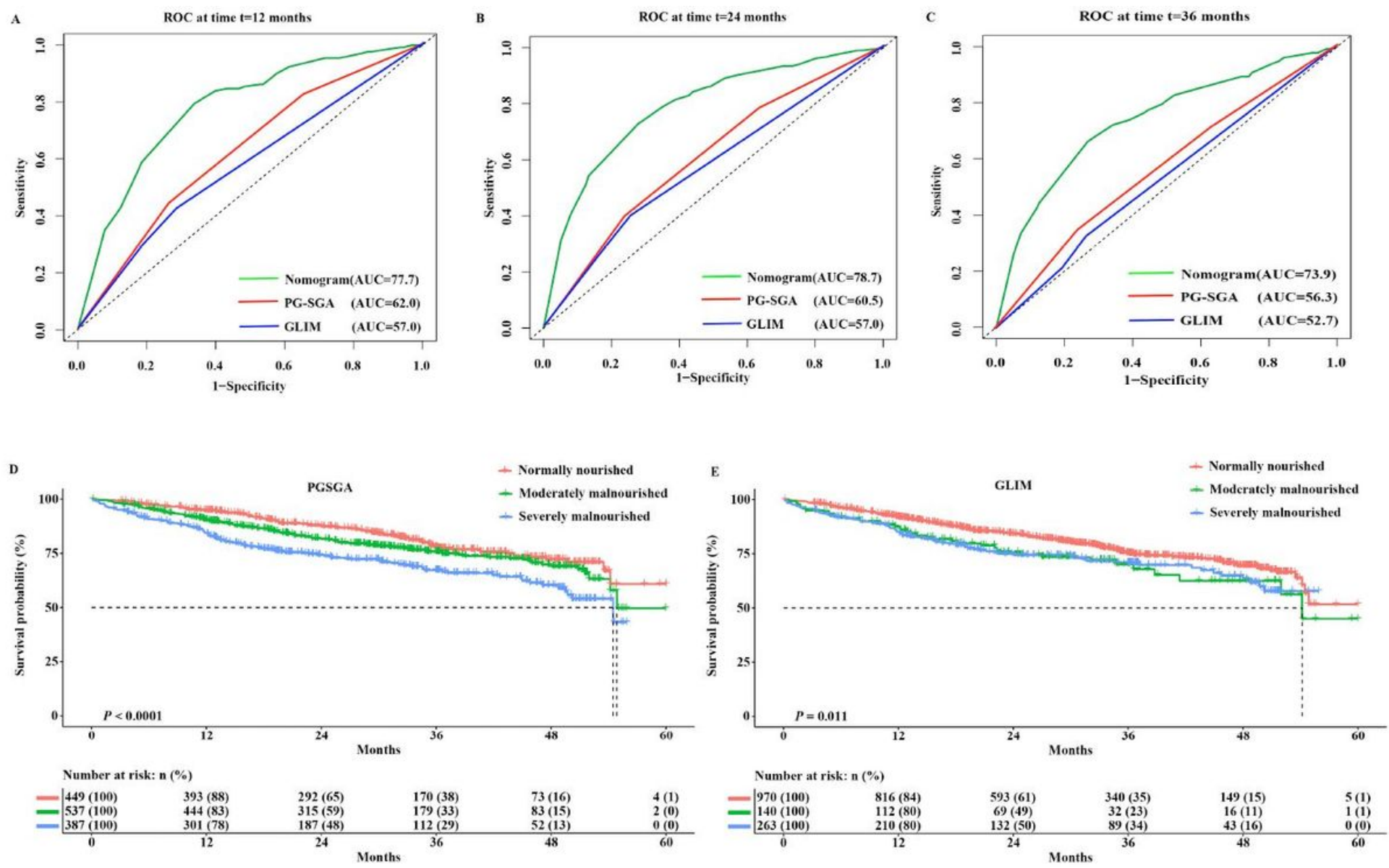

\section{Figure 4}

The 12-, 24-, and 36-month time-dependent ROC curves and survival curves of CRC OS. (A) ROC curves for 12-, 24-, and 36-month OS in the primary cohort. (B) Survival curve of PG-SGA in CRC OS; (C) Survival curve of GLIM in CRC OS. Notes: CRC: Colorectal cancer; OS: Overall survival; PG-SGA: Patient-Generated Subjective Global Assessment; GLIM: Global Leadership Initiative on Malnutrition.

A

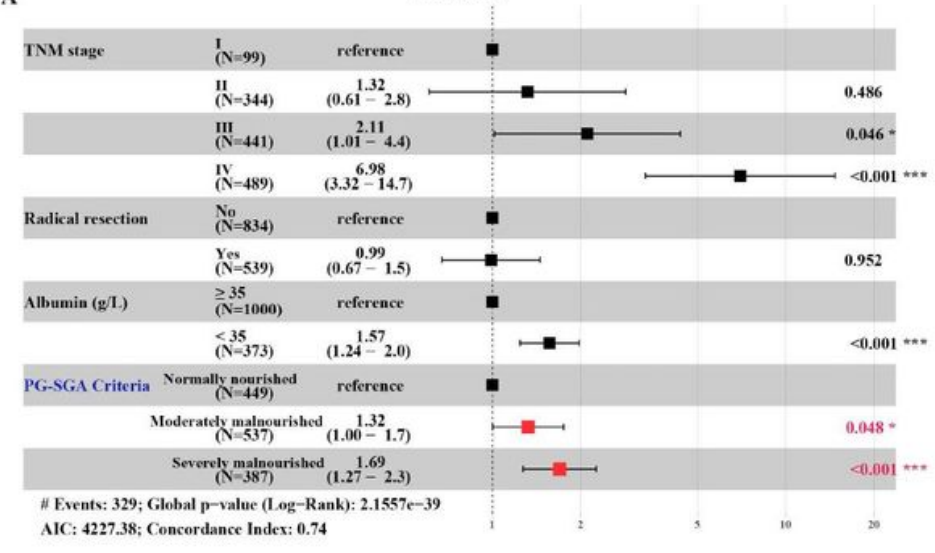

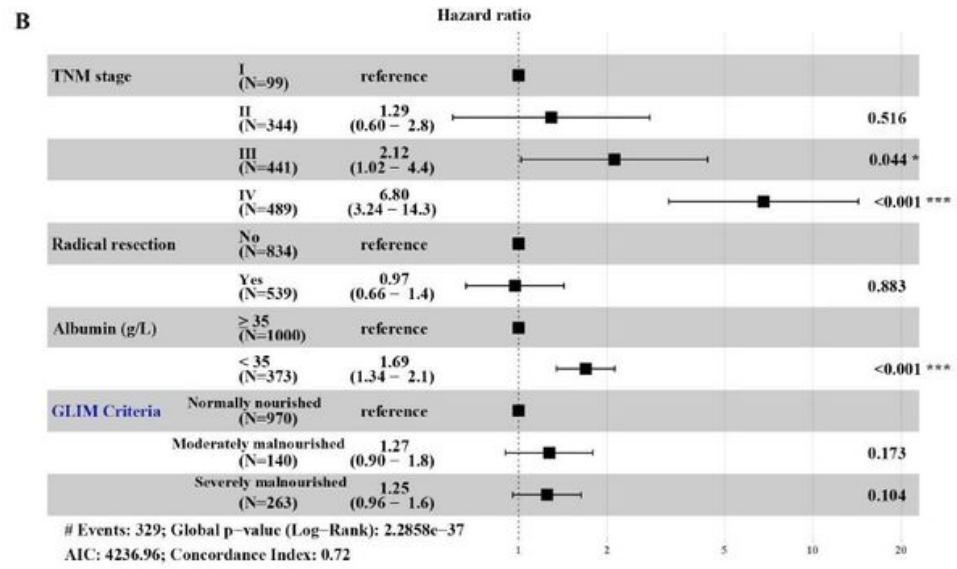

\section{Figure 5}

The forest plots of the PG-SGA and GLIM criteria after adjusting by nomogram model. (A) PG-SGA criteria; (B) GLIM criteria. Notes: TNM stage: Tumor-nodemetastasis stage; PG-SGA: Patient-Generated Subjective Global Assessment; GLIM: Global Leadership Initiative on Malnutrition. 
A

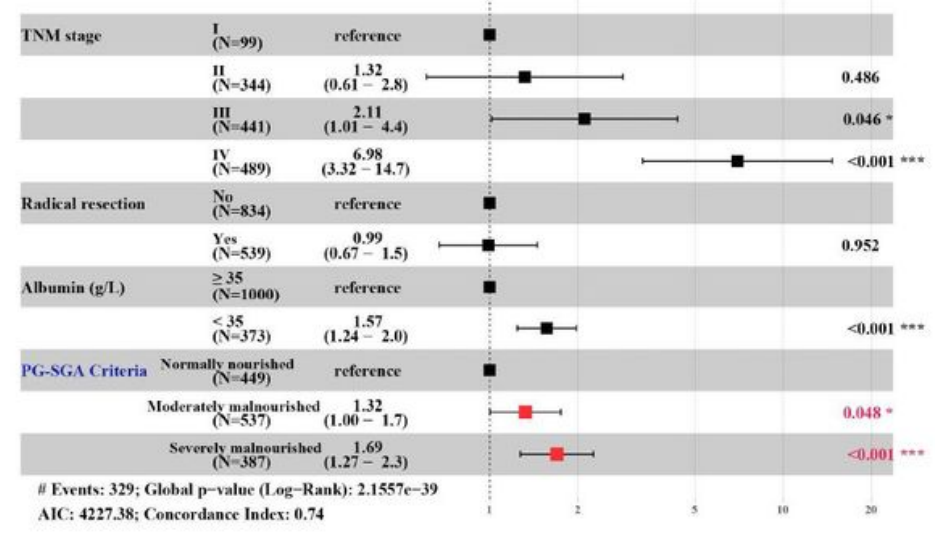

B

Hazard ratio

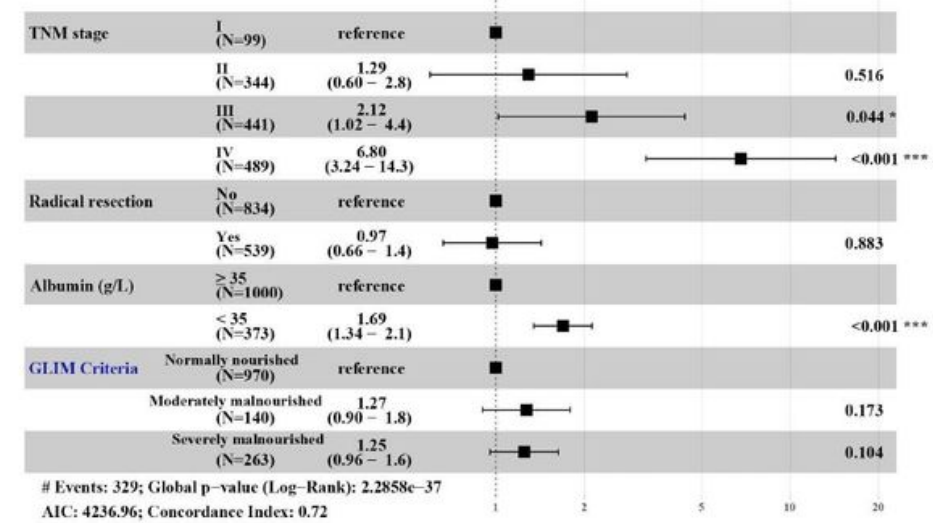

\section{Figure 5}

The forest plots of the PG-SGA and GLIM criteria after adjusting by nomogram model. (A) PG-SGA criteria; (B) GLIM criteria. Notes: TNM stage: Tumor-nodemetastasis stage; PG-SGA: Patient-Generated Subjective Global Assessment; GLIM: Global Leadership Initiative on Malnutrition.

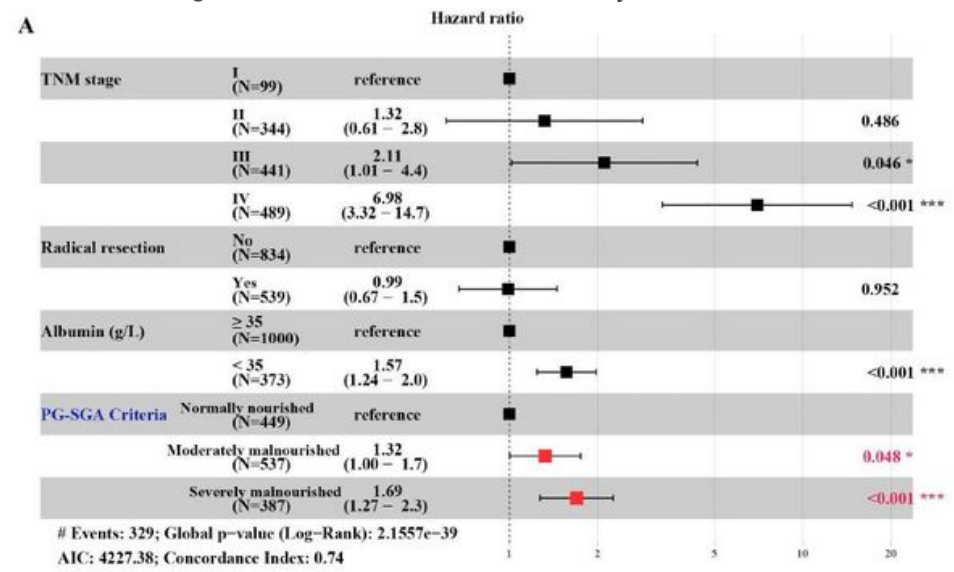

B

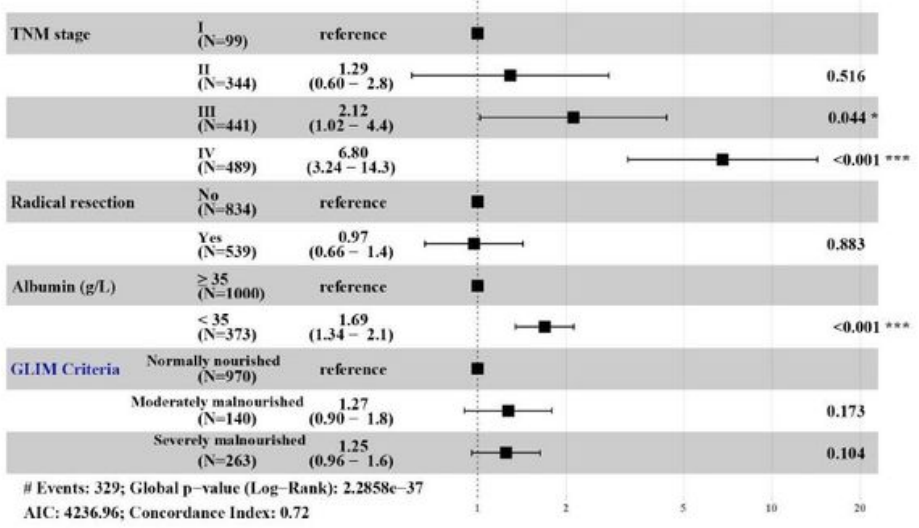

\section{Figure 5}

The forest plots of the PG-SGA and GLIM criteria after adjusting by nomogram model. (A) PG-SGA criteria; (B) GLIM criteria. Notes: TNM stage: Tumor-nodemetastasis stage; PG-SGA: Patient-Generated Subjective Global Assessment; GLIM: Global Leadership Initiative on Malnutrition.

PG-SGA

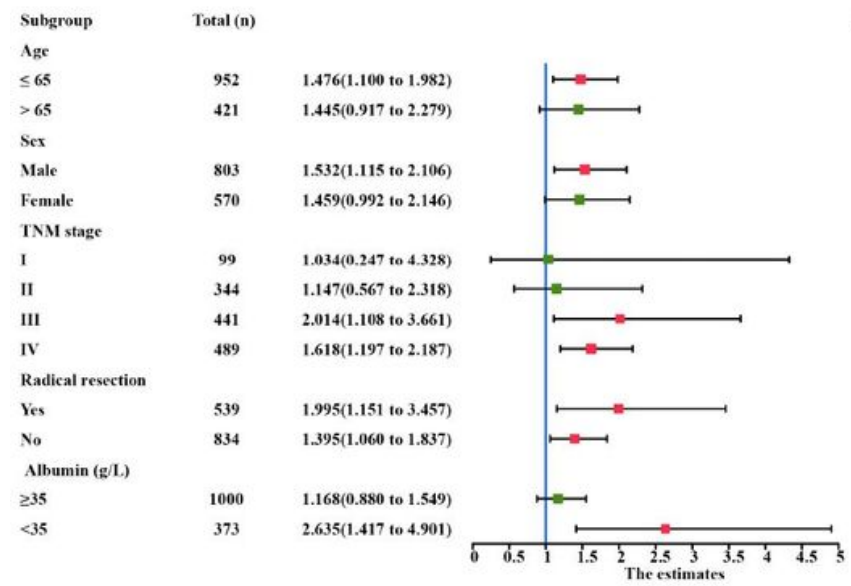

\section{Figure 6}

The stratification analysis of the PG-SGA and GLIM criteria in CRC OS.
GLIM

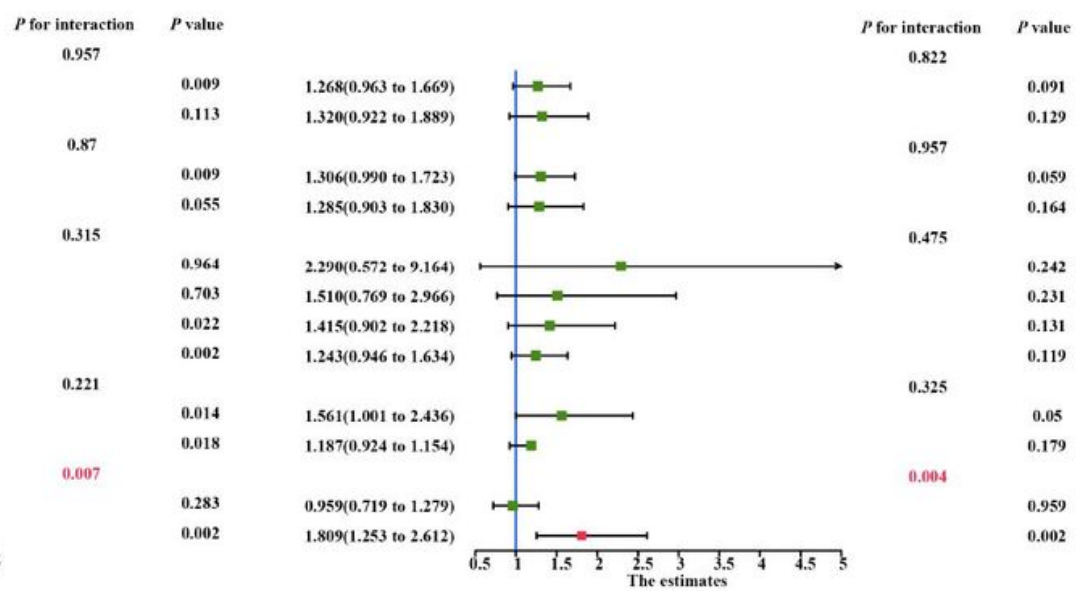



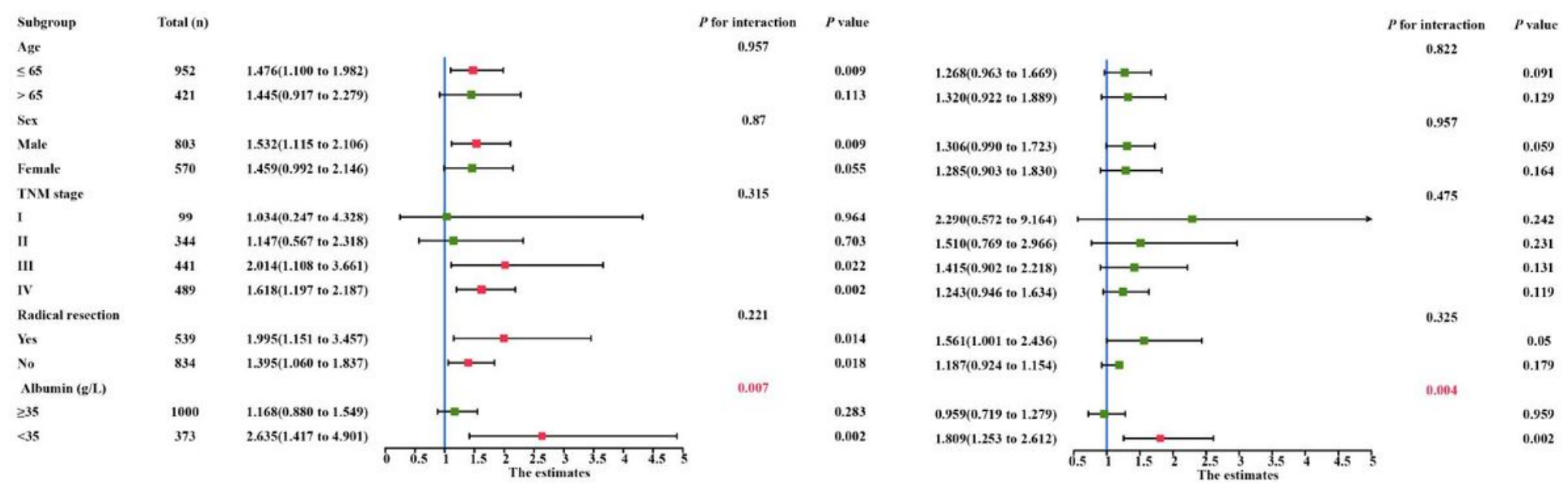

\section{Figure 6}

The stratification analysis of the PG-SGA and GLIM criteria in CRC OS.

GLIM
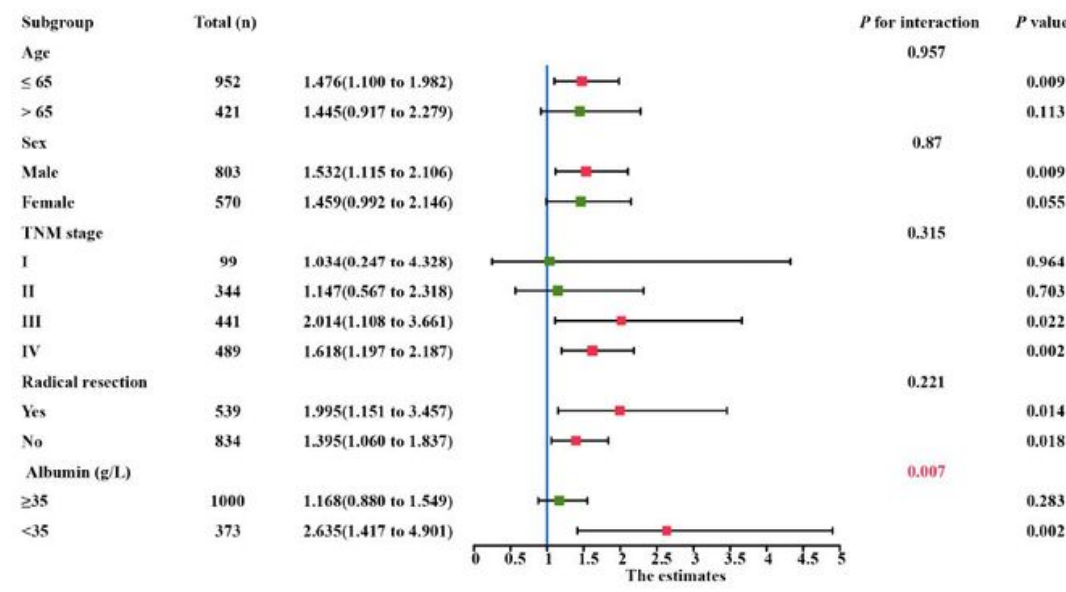

\section{Figure 6}

The stratification analysis of the PG-SGA and GLIM criteria in CRC OS.

\section{Supplementary Files}

This is a list of supplementary files associated with this preprint. Click to download.

- SupplementalTable.docx

- SupplementalTable.docx

- SupplementalTable.docx 